\title{
CORRELATIONS AND PAIRING BETWEEN ZEROS AND CRITICAL POINTS OF GAUSSIAN RANDOM POLYNOMIALS
}

\author{
BORIS HANIN
}

\begin{abstract}
We study the asymptotics of correlations and nearest neighbor spacings between zeros and holomorphic critical points of $p_{N}$, a degree $N$ Hermitian Gaussian random polynomial in the sense of Shiffman and Zeldtich, as $N$ goes to infinity. By holomorphic critical point we mean a solution to the equation $\frac{d}{d z} p_{N}(z)=0$. Our principal result is an explicit asymptotic formula for the local scaling limit of $E\left[Z_{p_{N}} \wedge C_{p_{N}}\right]$, the expected joint intensity of zeros and critical points, around any point on the Riemann sphere. Here $Z_{p_{N}}$ and $C_{p_{N}}$ are the currents of integration (i.e. counting measures) over the zeros and critical points of $p_{N}$, respectively. We prove that correlations between zeros and critical points are short range, decaying like $e^{-N|z-w|^{2}}$. With $|z-w|$ on the order of $N^{-1 / 2}$, however, $E\left[Z_{p_{N}} \wedge C_{p_{N}}\right](z, w)$ is sharply peaked near $z=w$, causing zeros and critical points to appear in rigid pairs. We compute tight bounds on the expected distance and angular dependence between a critical point and its paired zero.
\end{abstract}

\section{INTRODUCTION}

Let $p_{N}$ be a degree $N$ polynomial in one complex variable. We study in this paper how its zeros and holomorphic critical points (those $z$ for which $\frac{d}{d z} p_{N}(z)=0$ ) are correlated when $p_{N}$ is random and $N$ is large. To motivate the study of correlations between zeros and holomorphic critical points, we recall the following classical theorem from complex analysis.

Theorem (Gauss-Lucas). The holomorphic critical points of any polynomial in one complex variable are contained in the convex hull of its zeros.

Non-trivial correlations between zeros and critical points of random polynomials must therefore always exist. We prove in this paper that, at least for Hermitian Gaussian random polynomials in the sense of Bleher, Shiffman, and Zelditch in [1, 3, 15] (cf Section 1.1 for a definition), a zero of $p_{N}$ at $z$ and a holomorphic critical point of $p_{N}$ at $w$ are essentially uncorrelated unless $|z-w|$ is on the order of $N^{-1 / 2}$. This follows from Theorems 1 and 3 . On the $N^{-1 / 2}$ length-scaled, however, we find that, on average, zeros and critical points appear in rigid pairs (cf Figures 1-3). This statement is quantified in Theorems 1 and 2 .

We assume from now on that $p_{N}$ is a degree $N$ Hermitian Gaussian random polynomial. We associate to $p_{N}$ the currents of integration (equivalently counting measures)

$$
Z_{p_{N}}:=\sum_{p_{N}=0} \delta_{z} \quad \text { and } \quad C_{p_{N}}:=\sum_{\frac{d}{d w} p_{N}(w)=0} \delta_{w}
$$

over its zeros and holomorphic critical points and study the expected joint intensity

$$
K_{N}:=E\left[Z_{p_{N}} \wedge C_{p_{N}}\right] .
$$

We will refer to $K_{N}$ as the cross-correlation current. As in [1, 3, 15, 16] and elsewhere, our methods combine the Poincaré-Lelong formula with Szëgo kernel asymptotics in the setting of positive holomorphic line bundles over compact complex manifolds. 
This paper is the first to consider correlations and nearest neighbor spacings between zeros and holomorphic critical points of $p_{N}$. Critical points with respect to smooth metric connections were considered in [2, 4, 5, 11] and, as explained in Section 1.4, result in a significantly different theory. Perhaps the most striking difference is that zeros and holomorphic critical points are highly correlated and tend to appear in rigid pairs. This is illustrated in Figures 1-4 for $p_{50}$, a random degree 50 polynomial drawn from the computationally tractable $S U(2)$ ensemble described in Section 2.1. The colored lines in these figures are the (negative) gradient flow lines of $M(z):=\left|p_{50}(z)\right|^{2}$. Zeros and holomorphic critical points of $p_{50}$ are the local minima and saddle points of $M$, respectively. Flow lines terminating in a given zero or critical point are drawn in the same color.

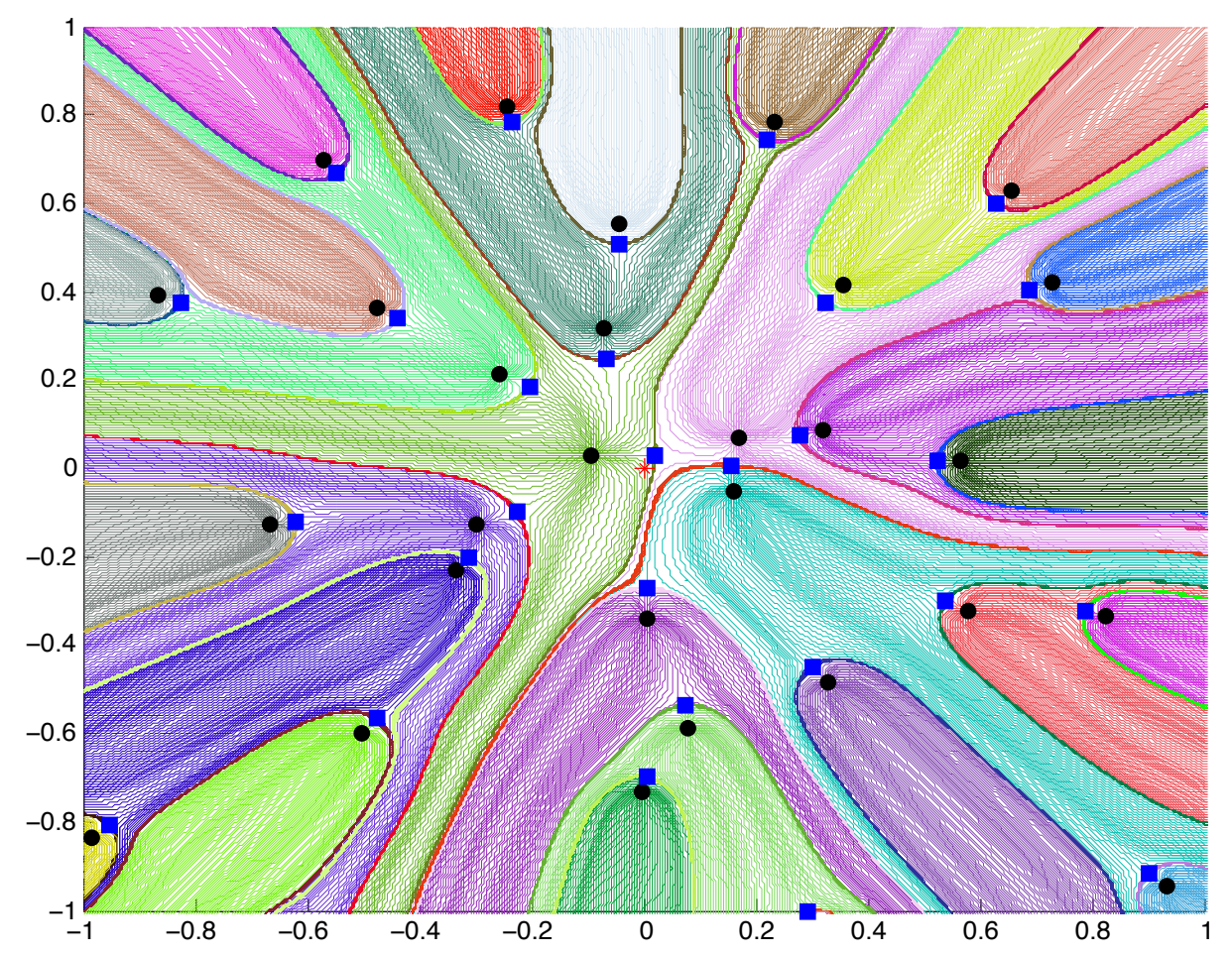

FiguRE 1. Zeros (black discs) and holomorphic critical points (blue squares) for an $S U(2)$ polynomial $p_{50}$ of degree 50 . The origin is denoted by a red asterisk.

$M(z)$ is subharmonic and so cannot have any local maxima. The basin of attraction for a given zero (i.e. those points in $\mathbb{C}$ whose gradient flow lines terminate in that zero) is therefore unbounded. By comparison, the basins of attraction considered by Nazarov, Sodin, and Volberg in [11] are compact and have constant area with probability 1. The difference is that while they study the zeros of a Gaussian Analytic Function $f$, the saddle points of their potential are critical points of the random smooth function $f(z) e^{-\frac{1}{2}|z|^{2}}$ rather than $f$ itself. Their critical points are therefore computed with respect to the metric connection of the hermitian metric $h(z)=\frac{1}{\pi} e^{-|z|^{2}}$ on the trivial line bundle $\mathbb{C} \times \mathbb{C} \rightarrow \mathbb{C}$. This paper investigates the purely holomorphic setting where, as explained in Section 1.4, we do not include the metric factor $e^{-\frac{1}{2}|z|^{2}}$ in contructing the potential. 
1.1. Definitions and Notation. Let $h$ be a smooth positive Hermitian metric on $\mathcal{O}(1) \rightarrow$ $\mathbb{C} P^{1}$. We recall the definition of the Hermitian Gaussian ensemble associated to $h$. Fix $N \geq 1$ and write $\mathcal{P}_{N}$ for the space of polynomials of degree at most $N$ in one complex variable. We identify $\mathcal{P}_{N}$ with $H_{\text {hol }}^{0}\left(\mathbb{C} P^{1}, \mathcal{O}(N)\right)$, the space of global sections of $\mathcal{O}(N) \rightarrow \mathbb{C} P^{1}$, by the linear map $z^{j} \mapsto z_{1}^{j} z_{0}^{N-j}$ (cf Section 2.3). A random polynomial (section) of degree $N$ drawn from this ensemble is

$$
p_{N}:=\sum_{j=0}^{N} a_{j} S_{j},
$$

where $a_{j} \sim N(0,1)_{\mathbb{C}}$ are i.i.d. standard complex Gaussians and $\left\{S_{j}\right\}_{j=0}^{N}$ is any orthonormal basis for $H_{\text {hol }}^{0}\left(\mathbb{C} P^{1}, \mathcal{O}(N)\right)$ with respect to the inner product

$$
\left\langle s_{1}, s_{2}\right\rangle_{h}:=\int_{\mathbb{C} P^{1}} h^{N}\left(s_{1}(z), s_{2}(z)\right) \omega_{h}(z), \quad s_{1}, s_{2} \in H_{h o l}^{0}\left(\mathbb{C} P^{1}, \mathcal{O}(N)\right) .
$$

Here $\omega_{h}:=\frac{i}{2 \pi} \partial \bar{\partial} \log h^{-2}$ is the first Chern class of $(\mathcal{O}(1), h)$. We say that $p_{N}$ is a random polynomial of degree $N$ drawn from the Hermitian Gaussian ensemble corresponding to $h$.

We denote throughout by $z_{0}$ the usual frame of $\mathcal{O}(1)$ over $\mathbb{C} P^{1} \backslash\{\infty\}$ (cf Section 2.3) and define $\nabla^{z_{0}}$ to be the meromorphic connection on $\mathcal{O}(1)$ for which $z_{0}$ is parallel (cf Section 4 ). Writing $p_{N}$ for a degree $N$ polynomial and the section it represents, the critical point equation $\frac{d}{d z} p_{N}(z)=0$ becomes $\nabla^{z_{0} \otimes N} p_{N}=0$. Relative to the frame $z_{0}, \nabla^{z_{0}}$ has an indentically zero connection 1 -form. Hence, it is related to the metric connection $\nabla^{h}$ of $h$ via

$$
\nabla^{z_{0}}=\nabla^{h}-\partial \phi_{z_{0}}
$$

where

$$
\phi_{z_{0}}(z):=\log \left\|z_{0}(z)\right\|_{h}^{-2} .
$$

The function $\phi_{z_{0}}$ will play an important role in our results.

1.2. Informal Discussion of Results. Our main result is Theorem 1. Together with Theorem 4, it gives an asymptotic formula for $K_{N}$ in local coordinates near any $\xi$ on the Riemann sphere. More precisely, we compactify $\mathbb{C}$ into the Riemann sphere $\mathbb{C} P^{1} \cong S^{2}$ and fix $\xi \in \mathbb{C} P^{1}$. To resolve individual zeros and critical points appearing near $\xi$ and study their correlations, we work in a particular holomorphic coordinate, called a Kähler normal coordinate, centered at $\xi$ and dilate by a factor of $N^{1 / 2}$ relative to $\xi$ (cf Definition 1 ). Our choice of coordinate is adapted to $h$ and gives a universal yardstick for measuring local correlations (cf Section 2.4). The $N^{1 / 2}$ scaling compensates for the typical $N^{-1 / 2}$ distance between $N$ well-spaced points on $\mathbb{C} P^{1}$.

Theorem 1 shows that correlations between zeros and critical points in scaled coordinates near $\xi$ depend strongly on whether $d \phi_{z_{0}}(\xi)=0\left(\phi_{z_{0}}\right.$ is defined in (1.3)). Namely, when $d \phi_{z_{0}}(\xi)=0$, zeros and critical points are highly correlated but stay a bounded distance apart and tend to appear in rigid pairs (cf Figure 2). When $d \phi_{z_{0}}(\xi) \neq 0$, however, zero and critical point pairs are separated by a distance of at most $N^{-1 / 4}$ and hence coincide in the large $N$ limit (see Figure 3). This happens because the leading term in powers of $N$ for the connection $\nabla^{z_{0} \otimes N}$ in scaled coordinates near such $\xi$ is an order 0 differential operator. Zeros and critical points thus become indistinguishable. This can be seen directly from (1.2) and is explained in Section 4 .

In Theorem 2, our main application of Theorem 1, we study nearest neighbor spacings between zeros and critical points in scaled local coordinates near a fixed $\xi \in \mathbb{C} P^{1}$. We fix a 


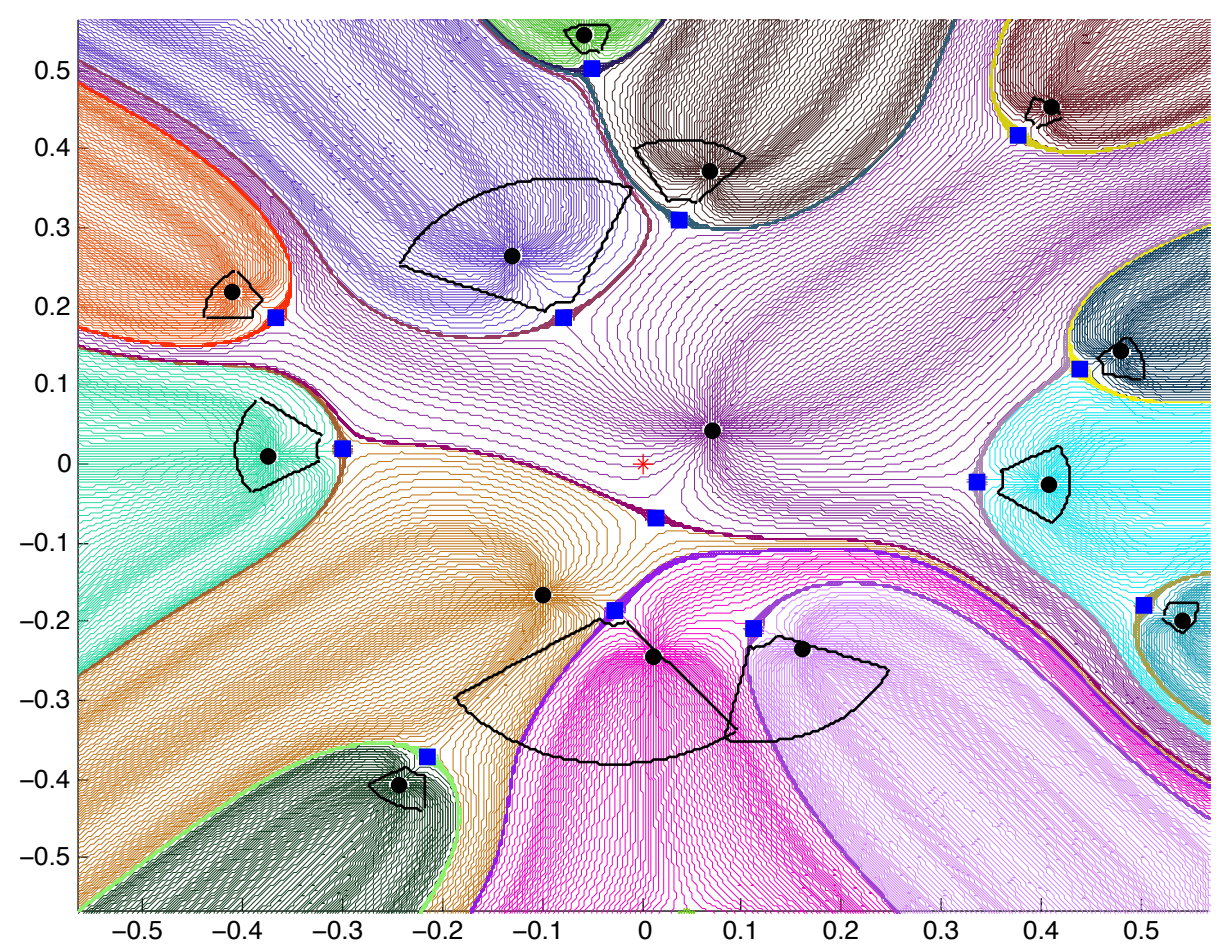

FiguRE 2. Zeros (black discs) and holomorphic critical points (blue squares) for an $S U(2)$ polynomial $p_{50}$ of degree 50 inside $[-4 / \sqrt{50}, 4 / \sqrt{50}]^{2}$ in Kähler normal coordinates around $\xi=[1: 0]$, the unique point for which $d \phi_{z_{0}}(\xi)=0$. Near each critical point at $w$ with $|w|>1$, the sector predicted in Theorem 2 (with parameter $c=3 / 4$ ) to contain its paired zero is shown.

measurable set $A$ in these coordinates and show that the expected number of critical points lying in $A$ is equal to the expected number of zero and critical point pairs $(z, w)$ with $w \in A$ and $z$ "paired" with $w$ is a nearly deterministic way. It is tempting to interpret this result by saying that, on average, each critical point comes paired with a unique zero. Although this interpreation is plausible from Figures 1-3, Theorem 2 is consistent with the possibilities such as, on average, half the critical points of $p_{N}$ being paired with two zeros and half are not being paired with any zeros. Developing the tools to exclude such possibilities is work in progress by the author.

In addition to the cross correlation current $K_{N}$, we fix $\xi \in \mathbb{C} P^{1}$ and treat in Theorem 3 the conditional current

$$
E\left[C_{p_{N}} \mid p_{N}(\xi)=0\right]
$$

in the sense of Shiffman, Zelditch, and Zhong in [16]. As explained in Section 6.1 of [16], $E\left[C_{p_{N}} \mid p_{N}(\xi)=0\right]$ gives a measure of the correlations between zeros and critical points that is quite different from the conditional density obtained from $K_{N}$. Finally, in Theorem 4, we give local and global asymptotics for the (unconditional) expected distribution of critical points $E\left[C_{p_{N}}\right]$.

1.3. Formal Statement of Results. To state our results, we introduce the covariance current

$$
\operatorname{Cov}_{N}:=E\left[C_{p_{N}} \wedge Z_{p_{N}}\right]-E\left[C_{p_{N}}\right] \wedge E\left[Z_{p_{N}}\right]
$$




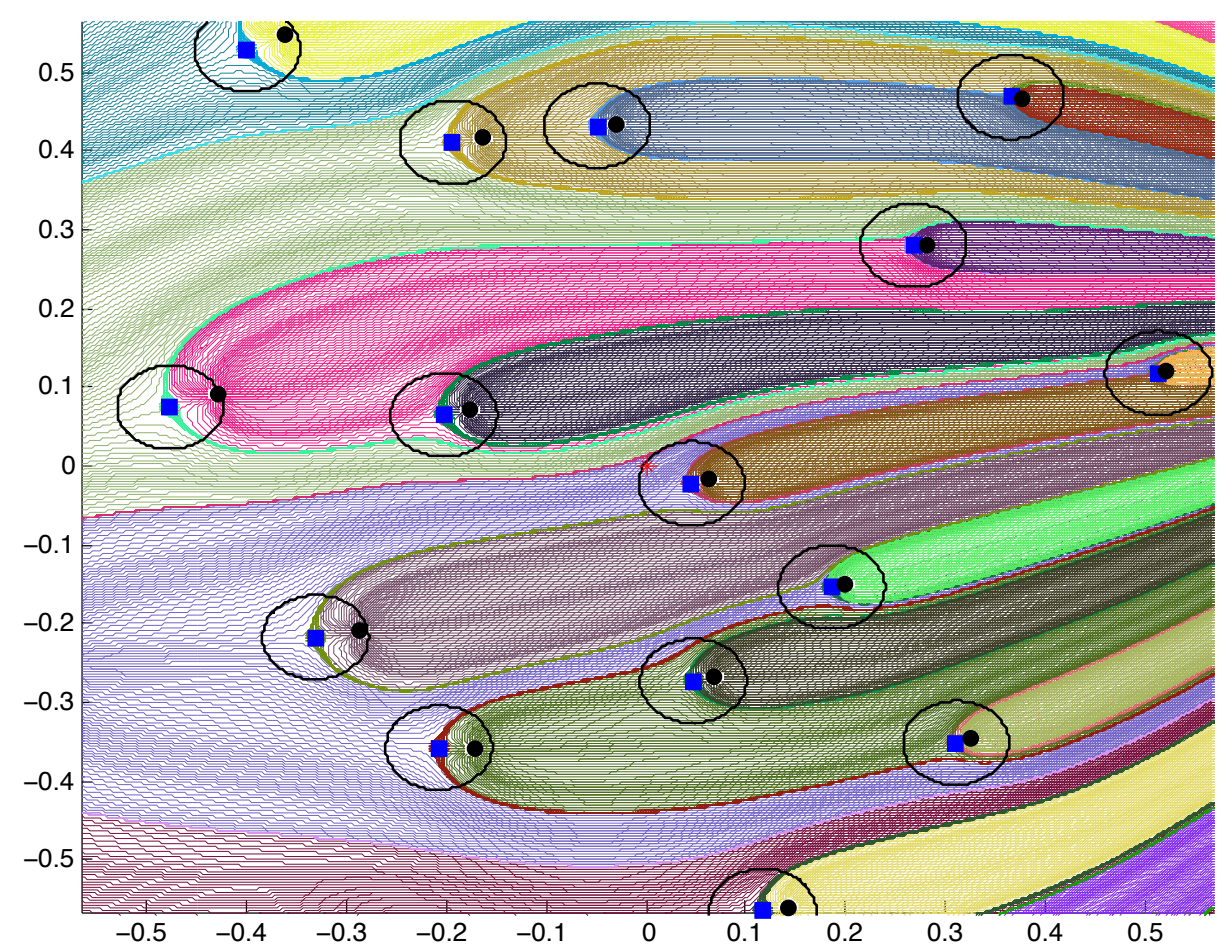

FiguRE 3. Zeros (black discs) and holomorphic critical points (blue squares) for an $S U(2)$ polynomial $p_{50}$ of degree 50 inside $[-4 / \sqrt{50}, 4 / \sqrt{50}]^{2}$ in Kähler normal coordinates around a generic point $\xi=2+.3 i$, which is shown as a red asterisk. Around each critical point, the circle of radius $N^{-3 / 4}$, predicted by Theorem 2 to contain its paired zero, is shown.

The currents $E\left[C_{p_{N}}\right]$ and $E\left[Z_{p_{N}}\right]$ are relatively simple (see Theorem 4 and Remark 5) so that the study of $K_{N}$ and $\operatorname{Cov}_{N}$ are essentially equivalent. We also define

$$
G(t):=\frac{\gamma}{4}-\frac{1}{4} \int_{0}^{t^{2}} \frac{\log (1-s)}{s} d s \quad t \in[0,1)
$$

where $\gamma$ is the Euler-Macheroni constant. Finally, let $\xi \in \mathbb{C} P^{1}$ and fix $N \geq 1$. Consider any neighborhood $U$ of $z$ and any holomorphic coordinate $z: U \rightarrow \mathbb{C}$ such that $z(\xi)=0$ and $\omega_{h}(z)=d z \wedge d \bar{z}+o\left(|z|^{2}\right)$. ( $z$ is called a Kähler normal coordinate and exists on any Kähler manifold). We make the following

Definition 1. We define $u: U \rightarrow \mathbb{C}$ given by

$$
u=u(z):=z \cdot N^{1 / 2}
$$

to be a $N^{-1 / 2}$ - scale normal coordinate at $\xi$.

Theorem 1 (Covariance Current Asymptotics). Let $h$ be a smooth positive Hermitian metric on $\mathcal{O}(1)$, and suppose $p_{N}$ is a degree $N$ polynomial drawn from the Hermitian Gaussian ensemble corresponding to $h$.

1. Global Asymptotics. For each $N \geq 1, \epsilon>0$ and every $\psi \in C^{4}\left(\mathbb{C} P^{1} \times \mathbb{C} P^{1}\right)$

$$
\left(\frac{1}{N(N-1)} \operatorname{Cov}_{N}(z, w), \psi(z, w)\right)=O\left(N^{-2+\epsilon}\right) .
$$


The implied constant depends only on $\epsilon$ and $\psi$.

2. Local Asymptotics. Fix $N \geq 1, \xi \in \mathbb{C} P^{1}$, and a $N^{-1 / 2}$-scale normal coordinate centered at $\xi$. For every $\epsilon>0$

$$
\operatorname{Cov}_{N}(z, w)=\left(\frac{i}{2 \pi} \partial_{z} \bar{\partial}_{z} \wedge \frac{i}{2 \pi} \partial_{w} \bar{\partial}_{w}\right) G\left(P_{\xi}(z, w)\right)+O\left(N^{-1 / 2+\epsilon}\right),
$$

where

$$
P_{\xi}(z, w):=\left\{\begin{array}{l}
e^{-\frac{1}{2}|z-w|^{2}}, \text { if } d \phi_{z_{0}}(\xi) \neq 0 \text { or } \xi=\infty \\
\frac{\left|\bar{w} \cdot \frac{\partial^{2} \phi_{z_{0}}}{\partial \bar{w}^{2}}\right|_{\xi}+z \mid}{\sqrt{1+\left|\bar{w} \cdot \frac{\partial^{2} \phi_{z_{0}}}{\partial \bar{w}^{2}}\right|_{\xi}+\left.w\right|^{2}}} e^{-\frac{1}{2}|z-w|^{2}}, \text { if } d \phi_{z_{0}}(\xi)=0
\end{array}\right.
$$

The implied constant in (1.5) depends on $\epsilon$, and the expression may be paired with any bounded measurable function.

Remark 1. If $d \phi_{z_{0}}(\xi) \neq 0$ or $\xi=\infty$, then our local formula for $\operatorname{Cov}_{N}$ is identical to the formula for variance current $E\left[Z_{p_{N}} \wedge Z_{p_{N}}\right]-E\left[Z_{p_{N}}\right] \wedge E\left[Z_{p_{N}}\right]$ derived in [15]. This may seem surprising. It is a consequence, as explained in Section 4, of the fact that the large $N$ limit of the derivative $\frac{d}{d z}$ in scaled coordinates near a point $\xi$ satisfying $d \phi_{z_{0}}(\xi) \neq 0$ or $\xi=\infty$ is an order 0 differential operator. Zeros and critical points therefore become indistinguishable. In contrast, when $d \phi_{z_{0}}(\xi)=0$, the scaling limit of $\frac{d}{d z}$ is a genuine order 1 differential operator.

Theorem 2, which we state next, is our main application of Theorem 1. Cases 1 and 2 are illustrated in Figures 3 and 2, respectively.

Theorem 2 (Expected Nearest Neighbor Spacings). With the notation of Theorem 1, fix $\xi \in \mathbb{C} P^{1}$ and consider a $N^{-1 / 2}$ - scale normal coordinate centered at $\xi$. Fix a bounded $A \subset \mathbb{C}$ and write

$$
C_{A}:=\#\left\{w \in A \mid \frac{d}{d w} p_{N}(w)=0\right\} \quad \text { and } \quad Z_{A}:=\#\left\{z \in A \mid p_{N}(z)=0\right\} .
$$

Case 1. Suppose that $d \phi_{z_{0}}(\xi) \neq 0$ or $\xi=\infty$. Define the random variables $\mathcal{X}_{A, N}$ to be the number of pairs $(z, w) \in \mathbb{C} \times \mathbb{C}$ such that

$$
w \in A, \quad p_{N}(z)=\frac{d}{d w} p_{N}(w)=0, \quad \text { and } \quad|z-w| \leq N^{-1 / 4} .
$$

Then, for each $\epsilon>0$,

$$
E\left[\mathcal{X}_{A, N}\right]=E\left[C_{A}\right]+O\left(N^{-1 / 4+\epsilon}\right)=E\left[Z_{A}\right]+O\left(N^{-1 / 4+\epsilon}\right) .
$$

Case 2. Suppose now that $d \phi_{z_{0}}(\xi)=0$. Define $\alpha:=\frac{\partial^{2} \phi_{z_{0}}}{\partial \bar{w}^{2}}(\xi)$ and introduce $\zeta:=\alpha \bar{w}+w$. Assume that $A \subseteq \mathbb{C} \backslash\{|\zeta| \leq 1\}$, and fix a parameter $c \in(2 / 3,1)$. Let $\mathcal{X}_{A, N, c}$ be the number of pairs $(z, w) \in \mathbb{C} \times \mathbb{C}$ such that

$$
w \in A, \quad p_{N}(z)=\frac{d}{d w} p_{N}(w)=0, \quad \text { and } \quad z=w+r e^{i t}
$$

with

$$
r \in\left[|\zeta|^{-1}-|\zeta|^{-1-c},|\zeta|^{-1}+|\zeta|^{-1-c}\right] \quad \text { and } \quad t \in\left[\arg (\zeta)-|\zeta|^{-c}, \arg (\zeta)+|\zeta|^{-c}\right] \text {. }
$$


We have for each $\epsilon>0$

$$
E\left[\mathcal{X}_{A, N, c}\right]=E\left[C_{A}\right]+O\left(\int_{A}|\zeta|^{2-3 c} d w \wedge d \bar{w}\right)+O\left(N^{-1 / 2+\epsilon}\right),
$$

where the implied constant in the first error term depends only on $c$ and in the second error term depends only on $\epsilon$.

Remark 2. The situation in Case 1 of Theorem 2 is the generic behavior. Indeed, the positivity of $h$ means that $\phi_{z_{0}}$ is subharmonic and so can vanish at only finitely many points in any bounded subset of $\mathbb{C} P^{1} \backslash\{\infty\}$. Moreover, $\phi_{z_{0}}$ must take the form $\log \left(1+|z|^{2}\right)+\psi(z)$ for some $\psi$ that is smooth function on all of $\mathbb{C} P^{1}$. Since the derivative of $\psi$ is bounded while the derivative of $\log \left(1+|z|^{2}\right)$ is unbounded at infinity, $d \phi_{z_{0}} \neq 0$ in some neighborhood of $\infty$.

Remark 3. In the case when $d \phi_{z_{0}}(\xi)=0$, the constrains (1.8) form a tight sector in polar coordinates around each critical point. The radial and angular widths of this sector decrease as $|w|$ grows and are determined by the 2 -jet of $\phi_{z_{0}}$ at $\xi$ via the paramter $\alpha$. Different values of $\alpha$ can cause rather different kinds of pairings. For example, $\alpha=0$ means $\zeta=w$, while $\alpha=1$ means $\zeta=\operatorname{Re} w$.

In addition to studying the covariance current $\operatorname{Cov}_{N}$, we study for any fixed $\xi \in \mathbb{C} P^{1}$ the conditional $(1,1)$-current

$$
E\left[C_{p_{N}} \mid p_{N}(\xi)=0\right]
$$

in the sense of Shiffman, Zelditch, and Zhong in [16]. Since the event $p_{N}(\xi)=0$ has probability 0 , we specify that the particular random variable used to define the conditional expectation is the evaluation map $e v_{\xi}$ at $\xi$. Continuing the trend of Theorems 1 and 2, we see in Theorem 3 that near $\xi$ satisfying $d \phi_{z_{0}}(\xi) \neq 0$ or $\xi=\infty$, the current $E\left[C_{p_{N}} \uparrow p_{N}(\xi)=0\right]$ is indistinguishable from the current $E\left[Z_{p_{N}} \mid p_{N}(\xi)=0\right]$ studied in [16]. Near $\xi$ satisfying $d \phi_{z_{0}}(\xi)=0$, however, a different behavior emerges.

Theorem 3. Let $h$ be a smooth positive Hermitian metric on $\mathcal{O}(1) \rightarrow \mathbb{C} P^{1}$ and suppose $p_{N}$ is a degree $N$ polynomial drawn from the Hermitian Gaussian ensemble corresponding to $h$. Fix $\xi \in \mathbb{C} P^{1}$.

1. Global Asymptotics. For each $N \geq 1$ and every $\psi \in C^{\infty}\left(\mathbb{C} P^{1}\right)$,

$$
\left(E\left[C_{p_{N}} \mid p_{N}(\xi)=0\right], \psi\right)=N \cdot\left(E\left[C_{p_{N}}\right], \psi\right)+O(1) .
$$

The implied constant is independent of $N$.

2. Local Asymptotics. Fix $\zeta \in \mathbb{C} P^{1}$ and a $N^{-1 / 2}$-scale normal coordinates centered at $\zeta$. If $\xi \neq \zeta$, then for each $k \geq 1$.

$$
E\left[C_{p_{N}} \mid p_{N}(\xi)=0\right](w)=E\left[C_{p_{N}}\right](w)+O\left(N^{-k}\right) .
$$

Suppose now $\xi=\zeta$. Then, if $d \phi_{z_{0}}(\xi) \neq 0$ or $\xi=\infty$, we have for any $\epsilon>0$

$$
E\left[C_{p_{N}} \mid p_{N}(\xi)=0\right](u)=E\left[C_{p_{N}}\right](u)+\frac{i}{2 \pi} \partial_{u} \bar{\partial}_{u} \log \left(1-e^{-|u|^{2}}\right)+O\left(N^{-1 / 2+\epsilon}\right) .
$$

Finally, if $\xi=\zeta$ and $d \phi_{z_{0}}(\xi)=0$, then $E\left[C_{p_{N}} \mid p_{N}(\xi)=0\right](u)$ is the smooth $(1,1)$-form

$$
\frac{i}{2 \pi} \partial_{u} \bar{\partial}_{u}\left(\log \left(1-P_{\xi}(u, 0)^{2}\right)+\log \left(1+\left|\frac{\partial^{2} \phi_{z_{0}}}{\partial u^{2}}\right|_{0} \cdot u+\left.\bar{u}\right|^{2}\right)\right)+O\left(N^{-1 / 2+\epsilon}\right),
$$


where, as in Theorem 1.

$$
P_{\xi}(u, 0)=\frac{\left|\bar{u} \cdot \frac{\partial^{2} \phi_{z_{0}}}{\partial \bar{u}^{2}}\right|_{\xi} \mid}{\sqrt{1+\left|\bar{u} \cdot \frac{\partial^{2} \phi_{z_{0}}}{\partial \bar{u}^{2}}\right|_{\xi}+\left.u\right|^{2}}} e^{-\frac{1}{2}|u|^{2}} .
$$

Remark 4. Expression (1.12) may be written explicitly as

$$
E\left[C_{p_{N}} \mid p_{N}(\xi)=0\right](u)=\pi \delta_{0}(u)+\left(\frac{1-\left(1+|u|^{2}\right) e^{-|u|^{2}}}{\left(1-e^{-|u|^{2}}\right)^{2}}+1\right) \frac{i}{2 \pi} \partial_{u} \bar{\partial}_{u}|u|^{2}+O\left(N^{-1 / 2+\epsilon}\right),
$$

and it coincides precisely with the expression obtained in [16] for $N^{-1 / 2}$ - scale current of $E\left[Z_{p_{N}} \mid p_{N}(\xi)=0\right]$. The reason is just as in Remark 1 .

Finally, we state our result about the local and global asymptotics for $E\left[C_{p_{N}}\right](w)$.

Theorem 4. Let $h$ be a smooth positive Hermitian metric on $\mathcal{O}(1) \rightarrow \mathbb{C} P^{1}$ and suppose $p_{N}$ is a degree $N$ polynomial drawn from the Hermitian Gaussian ensemble corresponding to $h$. 1. Global Asymptotics For each $N \geq 1$ and every $\psi \in C^{\infty}\left(\mathbb{C} P^{1}\right)$

$$
\left(E\left[C_{p_{N}}\right], \psi\right)=N \cdot\left(\omega_{h}, \psi\right)+O(1)
$$

with the implied constant depending only on $\psi$.

2. Local Asymptotics. Fix $\xi \in \mathbb{C} P^{1}$ and scaled normal coordinate centered at $\xi$. For each $N \geq 1$ we may write

$$
E\left[C_{p_{N}}\right](w)=\frac{1}{\pi} \partial \bar{\partial}|w|^{2}+O\left(N^{-1 / 2}\right) .
$$

if $d \phi_{z_{0}}(\xi) \neq 0$ or $\xi=\infty$. If, on the other hand, $d \phi_{z_{0}}(\xi)=0$, then

$$
E\left[C_{p_{N}}\right](w)=\frac{i}{2 \pi} \partial \bar{\partial} \log \left(1+\left|\frac{\partial^{2} \phi_{z_{0}}}{\partial w^{2}}\right|_{\xi} w+\left.\bar{w}\right|^{2}\right)+\frac{1}{\pi} \partial \bar{\partial}|w|^{2}+O\left(N^{-1 / 2}\right) .
$$

Both expressions (1.17) and (1.16) may be paired with any bounded measurable function.

Remark 5. It is an easy consequence of Lemma 3 below (and was proved as Lemma 3.1 in [12. for example) that in $N^{-1 / 2}$-scale normal coordinate centered at any $\xi \in \mathbb{C} P^{1}$, we have

$$
E\left[Z_{p_{N}}\right](z)=\frac{1}{\pi} \partial \bar{\partial}|z|^{2}+O\left(N^{-1 / 2}\right)
$$

which coincides with (1.16). The reason that zeros and critical points have the same expected distribution near $\xi$ satisfying $d \phi_{z_{0}}(\xi)=0$ or $\xi=\infty$ is the same as in Remark 1 .

1.4. Smooth Versus Holomorphic Critical Points. In order to put the current work in perspective, we contrast our purely holomorphic notion of critical points with the smooth critical points studied in [2, 4, 5, 11]. Let $p_{N}$ be a random polynomial of degree $N$ drawn from the Hermitian Gaussian ensemble corresponding to a fixed smooth positive hermitian metric $h$ on $\mathcal{O}(1)$.

As explained in Section 2.3, it is natural to view $p_{N}(z)$ as a holomorphic section of the line bundle $\mathcal{O}(N) \rightarrow \mathbb{C} P^{1}$. Critical points for sections of lines bundles depend on the choice of a connection. A natural choice of connection on $\mathcal{O}(N)$ is the $N^{t h}$ tensor power of $\nabla^{h}$, the metric connection compatible with $h$. Critical points with respect to $\nabla^{h}$ are what we refer to as 
smooth critical points. Holomorphic critical points correspond to the meromorphic connection $\nabla^{z_{0}}$ on $\mathcal{O}(1)$ defined in 1.2 ) (cf Section 4). Thus, while holomorphic critical points are solutions of $\frac{d}{d z} p_{N}(z)=0$, smooth critical points are solutions of $\frac{d}{d z}\left[p_{N}(z) \cdot\left\|z_{0}(z)\right\|_{h}^{-N}\right]=0$.

With respect to the usual frame $z_{0}$ of $\mathcal{O}(1)$ over $\mathbb{C} P^{1} \backslash\{\infty\}$ we deduce from equation (1.2)

$$
\nabla^{z_{0} \otimes N}=\nabla^{h}-N \cdot \partial \phi_{z_{0}} .
$$

The condition $d \phi_{z_{0}}(\xi) \neq 0$ implies that $\nabla^{z_{0}}$ becomes an order 0 operator to top order in $N$ locally around $\xi$. Zeros and critical points therefore become indistinguishable in the large $N$ limit. This accounts for the importance of the condition $d \phi_{z_{0}}(\xi)=0$ in Theorems 1,4 .

1.5. Acknowledgements. This work was suggested by Steve Zelditch, whose patient explanations about his work on Szëgo kernels and scaling limits for correlations of zeros have been invaluable. I would also like to thank Dean Baskin, Leonid Hanin, Eric Potash, Pokey Rule, Josh Shadlen, and Jared Wunsch for many useful discussions. Finally, I am indebted to Manjunath Krishnapur and Ron Peled for kindly sharing with me the Matlab code which I modified to produce the above figures.

1.6. Outline of Paper. The rest of the paper is organized as follows. First, in Sections 2.1 and 2.2, we introduce two model ensembles of Gaussian analytic functions that appear naturally in our work: the $S U(2)$ polynomials and the Bargmann-Fock random analytic functions. In Section 2.3, we recall some basic complex geometry and establish notation that will be used throughout. Next, in Section 2.4, we recall some notions from Kähler geometry and introduce the scaling limits of Theorems 1,4. We then recall in Section 3 how Szëgo kernels and their off-diagonal asymptotics are analyzed. We derive the scaling asymptotics for $\frac{d}{d z}$ in Section 4 and obtain as a consequence asymptotics for holomorphic derivatives of the Szëgo kernels in Section 5. The computations in this section are the extra ingedients needed to apply the methods of [1] and [15] to our problem. In Section 6, we recall and provide a proof of an important lemma that relates Szëgo kernels to the distribution of zeros and critical points for holomorphic sections of line bundles in general. Finally, in Sections 77, 10, we give proofs of our results.

\section{BACKGROUND}

We begin by introducing in Sections 2.1 and 2.2 two important and well-studied ensembles of random analytic functions: the $S U(2)$ polynomials and the Bargmann-Fock random analyltic functions.

2.1. $S U(2)$ Polynomials. The $S U(2)$ polynomials are the most computationally tractable of the Hermitian Gaussian ensembles. We introduce them for several reasons. First, we wish to derive the results of Theorem 4 without appealing to any difficult Szëgo Kernel asymptotics, which are necessary to treat the case of a general Hermitian Gaussian ensembles. Second, we wish to illustrate how the distribution of zeros and critical points near some $\xi \in \mathbb{C} P^{1}$ depends on whether $d \phi_{z_{0}}(\xi)$ vanishes. We mention that Feng and Wang in [7] study the distribution of critical values (rather than critical points) for $S U(2)$ polynomials.

In the language of our paper, $S U(2)$ polynomials are the Hermitian guassian ensemble corresponding to the Fubini-Study metric $h_{F S}$ on $\mathcal{O}(1)$. Over a point $\left[z_{0}: z_{1}\right] \in \mathbb{C} P^{1}$, the meric $h_{F S}\left(\left[z_{0}: z_{1}\right]\right)$ is obtained by restricting the usual Hermitian inner product on $\mathbb{C}^{2}$ to the complex line $\left.\mathcal{O}(-1)\right|_{\left[z_{0}: z_{1}\right]}$ and considering the dual metric. The name $S U(2)$ polynomials 
comes from the fact that $h_{F S}$ is invariant under the natural action of $S U(2)$ on $\mathbb{C} P^{1}$. We denote as in Section 2.3 by

$$
\left[z_{0}: z_{1}\right] \mapsto \frac{z_{1}}{z_{0}}=: z
$$

the standard affine coordinate on $\mathbb{C} P^{1}-\{\infty\}$ and by $z_{0}^{N}=z_{0}^{\otimes N}$ the standard frame for $\mathcal{O}(N)$ over $\mathbb{C} P^{1} \backslash\{\infty\}$. A degree $N S U(2)$ polynomial (section) is given explicitly by the formula

$$
p_{N}(z):=\left[\sum_{j=0}^{N} a_{j}\left((N+1)\left(\begin{array}{c}
N \\
j
\end{array}\right)\right)^{1 / 2} z^{j}\right] \cdot z_{0}^{N}, a_{j} \sim N(0,1)_{\mathbb{C}} \text { i.i.d. }
$$

The individual sections

$$
S_{j}(z):=\left((N+1)\left(\begin{array}{c}
N \\
j
\end{array}\right)\right)^{1 / 2} z^{j} \cdot z_{0}^{N}
$$

are orthonormal for the inner product (1.1). Indeed, in the coordinate $z$, this inner product may be written as follows:

$$
\left\langle s_{1}, s_{2}\right\rangle=\int_{\mathbb{C}} \frac{f_{1}(z) \overline{f_{2}(z)}}{\left(1+|z|^{2}\right)^{2 N+2}} \cdot \frac{i}{2 \pi} d z \wedge d \bar{z}, \quad s_{j}=f_{j} \cdot z_{0}^{N} \in H_{h o l}^{0}\left(\mathbb{C} P^{1}, \mathcal{O}(N)\right), j=1,2 .
$$

We have used that $h_{F S}(z)=\left(1+|z|^{2}\right)^{-1}$ so that

$$
\omega_{h}=\frac{i}{2 \pi} \partial_{z} \bar{\partial}_{z} \log h^{-2}=\frac{i d z \wedge d \bar{z}}{2 \pi\left(1+|z|^{2}\right)^{2}}
$$

and

$$
\left\|z_{0}\right\|_{h_{F S}}^{2 N}=\left(1+|z|^{2}\right)^{-2 N} .
$$

That the monomials $\left\{z^{j}\right\}_{j=0}^{N}$ are orthogonal follows immediately by passing to polar coordinates in $(2.2)$ and is true by the same argument for any toric metric that is equivariant with respect to the particular $S^{1}$ action on $\mathbb{C} P^{1}$ that fixes $[1: 0]$ and $[0: 1]$, the north and south poles. The $L^{2}$ norms

$$
\left\|z^{j}\right\|_{L^{2}}^{2}=\frac{1}{(N+1)\left(\begin{array}{c}
N \\
j
\end{array}\right)}
$$

may be computed by lifting $|z|^{2 j}$ to $S^{1}$ equivariant functions on the principle $S^{1}$ bundle $\pi: S^{3} \rightarrow \mathbb{C} P^{1}$ (the Hopf Fibration) and using that the Fubini-Study metric $\omega_{F S}$ on $\mathbb{C} P^{1}$ is the pushforward under $\pi$ of the round metric on $S^{3}$. Integration against the round metric on $S^{3}$ can then be further lifted to a guassian integral on $\mathbb{C}^{2}$. See Section 1.3 in [3] for more detials.

2.1.1. Expected Global Distribution of Zeros and Critical Point. Since $h_{F S}$ is invariant under the full $S U(2)$ group of isometries of $\left(\mathbb{C} P^{1}, \omega_{F S}\right)$, we immediately deduce that

$$
E\left[Z_{p_{N}}\right]=N \cdot \omega_{F S}
$$

We may see this alternatively from Lemma 2, which says that

$$
E\left[Z_{p_{N}}\right](z)=\frac{i}{2 \pi} \partial_{z} \bar{\partial}_{z} \log \left[(N+1) \sum_{j=0}^{N}\left(\begin{array}{c}
N \\
j
\end{array}\right)|z|^{2 j}\right]=\frac{i}{2 \pi} \partial_{z} \bar{\partial}_{z} \log \left[\left(1+|z|^{2}\right)^{N}\right] .
$$


Performing the differentiation yields

$$
E\left[Z_{p_{N}}\right](z)=\frac{N}{\pi}\left[\frac{1}{\left(1+|z|^{2}\right)^{2}}\right] \frac{i}{2} d z \wedge \overline{d z},
$$

as expected. Similarly, by Corollary 3 , we see that

$$
E\left[C_{p_{N}}\right](w)=\frac{i}{2 \pi} \partial_{w} \bar{\partial}_{w} \log \left[\frac{\partial^{2}}{\partial w \partial \bar{w}}\left(1+|w|^{2}\right)^{N}\right] .
$$

Again, we may perform the differentiation explicity to obtain

$$
E\left[C_{p_{N}}\right](w)=\frac{N}{\pi}\left[\left(\left(1-\frac{2}{N}\right) \frac{1}{\left(1+|w|^{2}\right)^{2}}+\frac{1}{\left(1+N|w|^{2}\right)^{2}}\right)\right] \frac{i}{2} d w \wedge \overline{d w} .
$$

Equation (2.4) recovers the results of Macdonald in [9]. The expected distribution of the zeros of $p_{N}$ is $N$ times Fubini-Study measure on $\mathbb{C} P^{1}$ for every N. In contrast, the critical points are only distributed uniformly on $\mathbb{C} P^{1}$ in the large $N$ limit. This is not surprising in light of the Guass-Lucas Theorem, which asserts that the holomorphic critical points of any complex polynomial lie inside the convex hull of its zeros.

2.1.2. Local Distribution of Zeros and Critical Points. We study the local behavior of zeros and critical points near some $\xi \in \mathbb{C} P^{1}$ in the $N^{-1 / 2}$-scale normal coordinates around $\xi$ of Definition 1 (cf also Section 2.4). The curvature of $h_{F S}$ is $\omega_{F S}$, the Fubini-Study metric on $\mathbb{C} P^{1}$. Kähler normal coordinates at $\xi=\left[\alpha_{0}: \alpha_{1}\right] \in \mathbb{C} P^{1}$ for $\omega_{F S}$ are given by the usual affine coordinate on $\left.\mathcal{O}(1)\right|_{\mathbb{C} P^{1}-\left\{-\bar{\alpha}_{1}: \overline{\alpha_{0}}\right\}}$. For example, in the standard (affine) coordinate centered at $[1: 0] \in \mathbb{C} P^{1}$ and with respect to the standard frame $z_{0}$ of $\mathcal{O}(1)$, we have that

$$
\omega_{F S}(z)=\partial \bar{\partial} \log \left\|z_{0}\right\|_{h_{F S}}^{-2}(z)=\partial \bar{\partial} \log \left(1+|z|^{2}\right)=d z \wedge d \bar{z}+o\left(|z|^{2}\right),
$$

as required. Fix some $\xi=\left[\xi_{0}: \xi_{1}\right] \neq[0: 1]$. Kähler normal coordinates around $\xi$ are given by

$$
\zeta:\left[z_{0}: z_{1}\right] \mapsto \frac{\xi_{0} z_{1}-\xi_{1} z_{0}}{\overline{\xi_{1}} z_{1}+\overline{\xi_{0}} z_{0}}
$$

the ratio of the sections of $\mathcal{O}(1)$ that vanish to order 1 at $\left[\xi_{0}: \xi_{1}\right]$ and at the antipodal point $\left[-\overline{\xi_{1}}: \overline{\xi_{0}}\right]$. The $N^{-1 / 2}$-scale Kähler normal coordinates of limits of Theorems 1 , 4 around $\xi=\left[\xi_{0}: \xi_{1}\right]$ are then obtained by rescaling

$$
\tau_{N}^{\xi}(\zeta):=\zeta \cdot N^{1 / 2}
$$

We see by direct computation from 2.3 that

$$
E\left[\left(\tau_{N}^{\xi}\right)_{*} Z_{p_{N}}\right](u) \rightarrow \partial_{u} \bar{\partial}_{u} \frac{i}{2 \pi}|u|^{2},
$$

in accordance with Remark 5. Similarly, from (2.4), we find that

$$
E\left[\left(\tau_{N}^{\xi}\right)_{*} C_{p_{N}}\right](u) \rightarrow \frac{i}{2 \pi} \partial_{u} \bar{\partial}_{u}\left(\log \left(1+|u|^{2}\right)+|u|^{2}\right)
$$

if $\xi=[1: 0]$ and

$$
E\left[\left(\tau_{N}^{\xi}\right)_{*} C_{p_{N}}\right](u) \rightarrow \partial_{u} \bar{\partial}_{u} \frac{i}{2 \pi}|u|^{2}
$$

otherwise. Since $d \phi_{z_{0}}(\xi)=0$ for the Fubini-Study metric if and only if $\xi=0$, this recovers the local asymptotics of $E\left[C_{p_{N}}\right]$ from Theorem 4 for special case of $h=h_{F S}$. 
2.2. Bargmann-Fock. The Bargmann-Fock Space $\mathcal{F}:=L^{2}\left(\mathbb{C}, \frac{1}{\pi} e^{-|z|^{2}} d z\right) \cap \mathcal{O}_{\text {hol }}(\mathbb{C})$ consists of the entire functions that are square integrable with respect to the standard gaussian measure $\frac{1}{\pi} e^{-|z|^{2}} d z$ on $\mathbb{C}$. An orthonormal basis for $\mathcal{F}$ with respect to the induced $L^{2}$ inner product is $\left\{\frac{z^{j}}{\sqrt{j !}}\right\}_{j \geq 0}$ and a Bargmann-Fock random analytic function, sometimes referred to as a Gaussian Entire Function or a Gaussian Analytic Function, is

$$
f(z):=\sum_{j=0}^{N} a_{j} \frac{z^{j}}{\sqrt{j !}}, \quad a_{j} \sim N(0,1)_{\mathbb{C}} \text { i.i.d }
$$

The Bargmann-Fock random analytic functions have been extensively studied in [8, 10, 11]. In the context of our work, we think of $\mathcal{F}$ as the space of $L^{2}$ holomorphic sections of the trivial line bundle $\mathbb{C} \times \mathbb{C}$ endowed with the Hermitian metic

$$
\|(z, 1)\|_{h_{B F}}^{2}:=\frac{1}{\pi} e^{-|z|^{2}} .
$$

Here $(z, 1)$ is the constant trivializing section of $\mathbb{C} \times \mathbb{C}$. The $L^{2}$ inner product on $\mathcal{F}$ is then

$$
\left\langle s_{1}, s_{2}\right\rangle=\int_{\mathbb{C}} h_{B F}\left(s_{1}(z), s_{2}(z)\right) w_{h_{B F}}(z)
$$

in complete analogy with 1.1 .

It is an important observation that the Bargmann-Fock ensemble is the local scaling limit for all the Hermitian Gaussian ensembles. To see this, we choose $\xi \in \mathbb{C} P^{1}$ and a $N^{-1 / 2}$-scale normal coordinate centered at $\xi$. As explained in Section 2.4, the Kähler potential for the metric $h^{N}$ then takes the form

$$
|z|^{2}+O\left(N^{-1 / 2}\right)
$$

which coincides to leading order with the potential for $h_{B F}$. Moreover, thought of as a holomorphic Gaussian field, $f$ is characterized by its covariance kernel

$$
\operatorname{Cov}_{B F}(z, w)=E\left[\|f(z) \otimes \overline{f(w)}\|_{h_{B F}}\right]=e^{z \cdot \bar{w}-\frac{1}{2}\left(|z|^{2}+|w|^{2}\right)} .
$$

Part of the content of the $C^{\infty}$ asymptotic expansion for the Szëgo kernels (see Section 3 ) is that in $N^{-1 / 2}$-scale Kähler normal coordinates around any $\xi \in \mathbb{C} P^{1}$, the covariance kernels

$$
\Pi_{N}(z, w)=E\left[\left\|p_{N}(z) \otimes \overline{p_{N}}(w)\right\|_{h^{N}}\right]
$$

for Hermitian Gaussian random sections of $\mathcal{O}(N)$ converge in the $C^{k}$ topology to $\operatorname{Cov}_{B F}(z, w)$ for all $k$. One may see this concretely for $S U(2)$ polynomials by applying Sterling's formula to $\left(\begin{array}{c}N \\ j\end{array}\right)$ in equation 2.1 and rescaling $z \mapsto \frac{z}{\sqrt{N}}$ to obtain

$$
\sum_{j=0}^{N} a_{j} \sqrt{(N+1)\left(\begin{array}{c}
N \\
j
\end{array}\right)}\left(\frac{z}{\sqrt{N}}\right)^{j} \approx \sum_{j=0}^{N} a_{j} \frac{z^{j}}{\sqrt{j !}},
$$

which is the truncated Bargmann-Fock random analytic function. 
2.3. Complex Projective Space. We recall some basic facts about $\mathbb{C} P^{1}$ and introduce some notation. By definition, $\mathbb{C} P^{1}$ is the space of complex lines through the origin in $\mathbb{C}^{2}$. Each line is determined by a pair $\left(z_{0}, z_{1}\right) \in \mathbb{C}^{1} \backslash\{(0,0)\}$. We denote by $\left[z_{0}: z_{1}\right]$ the equivalence class of pairs $\left(z_{0}, z_{1}\right)$ that determine the same line. The notation $\left[z_{0}: z_{1}\right]$ is called homogenous coordinates. We will refer to $[1: 0]$ and $[0: 1]$ variously as the south and north poles or at 0 and $\infty$, respectively.

The tautological line bundle $\mathcal{O}(-1) \rightarrow \mathbb{C} P^{1}$ assigns to each $\left[z_{0}: z_{1}\right]$ the line in $\mathbb{C}^{2}$ passing through $\left(z_{0}, z_{1}\right)$ and the origin. The total space of $\mathcal{O}(-1)$ is therefore $\mathbb{C}^{2}$ with the origin blown up. Every non-trivial holomorphic line bundle on $\mathbb{C} P^{1}$ is holomorphically isomorphic to a positice tensor power of either $\mathcal{O}(-1)$ and or its dual $\mathcal{O}(1)$. The line bundles

$$
\mathcal{O}(N):=\mathcal{O}(1)^{\otimes N}
$$

for $N \geq 1$ have an $N+1$-complex dimensional space of global sections. We denote this space by $H_{\text {hol }}^{0}\left(\mathbb{C} P^{1}, \mathcal{O}(N)\right)$. We write abusing notation $z_{0}$ and $z_{1}$ for the two global sections of $\mathcal{O}(1)$ that correspond to the linear functionals on $\mathbb{C}^{2}$ given by projection onto the first and second factors. Therefore,

$$
H_{h o l}^{0}\left(\mathbb{C} P^{1}, \mathcal{O}(N)\right)=\operatorname{Sym}^{N}\left(z_{0}, z_{1}\right),
$$

the space of symmetric polynomials in two complex variables. By the standard coordinate around $0=[1: 0] \in \mathbb{C} P^{1}$, we mean the coordinate $\left[z_{0}: z_{1}\right] \mapsto \frac{z_{1}}{z_{0}}=: z$ on $\mathbb{C} P^{1} \backslash\{[0: 1]\}$. Relative to the frame $z_{0}^{N}:=z_{0}^{\otimes N}$, every holomorphic section of $\mathcal{O}(N)$ is represented by a complex polynomial of degree $N$ :

$$
\sum_{j=0}^{N} a_{j} z_{1}^{j} z_{0}^{N-j}=\left(\sum_{j=0}^{N} a_{j} z^{j}\right) \cdot z_{0}^{N} .
$$

The map $p_{N}(z) \mapsto p_{N}(z) \cdot z_{0}^{N}$ is what we mean by identifying polynomials of degree $N$ is one complex variable with holomorphic sections of $\mathcal{O}(N)$ "in the usual way."

2.4. Scaling Limit and Kähler normal Coordinates. A compelling argument for studying local correlations between zeros and critical points in the $N^{-1 / 2}$-scale normal coordinates (see Definition 1) is the following. Given any positive line bundle $(L, h) \rightarrow M$ over a complex manifold and any $\xi \in M$, we may take $N^{-1 / 2}$-scale normal coordinates centered at $\xi$. In these coordinates $(L, h) \rightarrow M$ "converges" to line bundle $\mathbb{C} \times \mathbb{C}^{\operatorname{dim} M} \rightarrow M$ with its standard

Kähler metric $h=\frac{1}{\pi^{\operatorname{dim} M}} e^{-\frac{1}{2}\|z\|^{2}}$. More precisely, if $\phi$ a Kähler potential for $\omega_{h}$, then $N \phi$ is a Kähler potential for $\omega_{h^{N}}=N \cdot \omega_{h}$ and in a scaled normal coordinate centered at $\xi \in M$ for $\omega_{h}$, we have

$$
N \cdot \phi(z, \bar{z})=|z|^{2}+O\left(N^{-1 / 2}\right) .
$$

The leading term is precisely the Kähler potential for $\mathbb{C} \times \mathbb{C}^{\operatorname{dim} M}$. The choice of $N^{-1 / 2}$-scale normal coordinates therefore gives a kind of universal yardstick for studying the local correlations of zeros and critical points of random polynomials and, more generally, random section of positive line bundles.

\section{SzËGO KeRNELS}

Suppose $p_{N}$ is drawn from the Hermitian Guassian ensemble corresponding to a positive smooth Hermitian metric $h$ on $\mathcal{O}(1)$. Viewed as a Gaussian random field, its law and hence the joint statistics of its zero and critical point processes are determined by the its covariance 
kernel $\Pi_{N}$, the Szëgo Kernel associated to $\left(\mathcal{O}(N), h^{N}\right)$. Our main technical tool is therefore the $C^{\infty}$ complete asymptotic expansion for $\Pi_{N}$ of Shiffman and Zelditch given in [13, 15. We first recall the definition of the kernels $\Pi_{N}$ (Section 3.1) and introduce the related normalized Szëgo kernels (Section 3.2). Then, in Section 3.3, we recall the principle $S^{1}$ bundle $X \rightarrow \mathbb{C} P^{1}$ associated to $(\mathcal{O}(1), h) \rightarrow \mathbb{C} P^{1}$. The Szëgo kernels are most naturally analyzed by lifting to $X$. Finally, we use Section 3.4 to recall the relevant asymptotic expansions of $\Pi_{N}$ from [13].

3.1. Definition. Let $p_{N}=\sum_{j=0}^{N} a_{j} S_{j}$ be a Gaussian random polynomial (section of $\mathcal{O}(N)$ ) drawn from the Hermitian Gaussian Ensemble corresponding to a fixed smooth positive Hermitian metric on $\mathcal{O}(1)$. Its covariance kernel is called the Szëgo Kernel for $\left(\mathcal{O}(N), h^{\otimes N}\right)$ :

$$
\Pi_{N}(z, w):=\operatorname{Cov}\left(p_{N}(z), p_{N}(w)\right)=\sum_{j=0}^{N} S_{N}^{j}(z) \otimes \overline{S_{N}^{j}(w)} \in H_{h o l}^{0}\left(\mathbb{C} P^{1}, \mathcal{O}(N) \otimes \overline{\mathcal{O}(N)}\right),
$$

See the Introduction in [13] for details. The family of Szëgo kernels $\Pi_{N}$ is well-understood in the general setting of a positive holomorphic line bundle $(L, h) \rightarrow M$ over a compact complex manifold $M$ (cf [13, 15]).

3.2. Normalized Szëgo Kernel. As in [15, 16], it will be important to consider the Normalized Szëgo kernels:

$$
P_{N}(z, w):=\frac{\left\|\Pi_{N}(z, w)\right\|_{h^{N}}}{\sqrt{\left\|\Pi_{N}(z, z)\right\|_{h^{N}}\left\|\Pi_{N}(w, w)\right\|_{h^{N}}}}
$$

and

$$
\widetilde{P}_{N}(z, w):=\frac{\left\|1 \otimes \overline{\nabla_{V}^{z_{0}}} \Pi_{N}(z, w)\right\|_{h^{N}}}{\sqrt{\left\|\Pi_{N}(z, z)\right\|_{h^{N}}\left\|\nabla_{V}^{z_{0}} \otimes \overline{\nabla_{V}^{z_{0}}} \Pi_{N}(w, w)\right\|_{h^{N}}}} .
$$

We've written $V$ for an auxiliary non-vanishing local holomorphic vector field on which the value of $\widetilde{P}_{N}$ does not depend, and we've denoted as in Section 4 by $\nabla^{z_{0}}$ the meromorphic connection on $\mathcal{O}(N)$ that extends the holomorphic derivative $\frac{d}{d z}$. Perhaps to most natural reason to consider $P_{N}$ and $\widetilde{P}_{N}$ is probabilistic. Namely, $P_{N}(z, w)$ is the correlation between $p_{N}(z)$ and $p_{N}(w)$ and $\widetilde{P}_{N}(z, w)$ is the correlation between $p_{N}(z)$ and its derivative $\nabla_{V}^{z_{0}} p_{N}$.

3.3. Princple $S^{1}$ Bundle. Consider a positive line bundle $(L, h) \rightarrow M$ over a compact Käher manifold and an orthonormal basis $\left\{S_{j}\right\}_{j=0}^{d_{N}}$ for $H_{h o l}^{0}\left(L^{N}\right)$ with respect to the inner product (1.1). The $N^{\text {th }}$ Szëgo Kernel

$$
\Pi_{N}(z, w)=\sum_{j=0}^{d_{N}} S_{j}(z) \otimes \overline{S_{j}(w)}
$$

is studied in [13] by lifting sections $s \in H_{h o l}^{0}\left(M, L^{\otimes N}\right)$ to $S^{1}$-equivariant functions on the principle $S^{1}$ bundle associated to $(L, h)$. More precisely, we write $h^{*}$ for the dual metric on the dual bundle $L^{*}$ and define $X \rightarrow M$ by

$$
X:=\left\{v \in L^{*} \mid\|v\|_{h^{*}}=1\right\} .
$$


We denote by $\widehat{s}$ the lift of a section $s$ to the function $\widehat{s}(v):=v^{\otimes N}(s)$ on $X$. Writing $s=f \cdot e^{\otimes N}$ for local frame $e$ of $L$, we may write

$$
\widehat{s}(\theta, z):=e^{i N \theta}\|e(z)\|_{h}^{N} \cdot f(z) .
$$

Observe that

$$
|\widehat{s}(\theta, z)|=\|s(z)\|_{h^{N}} .
$$

The lifted Szëgo Kernel is then $\widehat{\Pi}_{N}(\alpha, z ; \beta, w)=\sum_{j=0}^{N} \widehat{S}_{j}(\alpha, z) \overline{\widehat{S}_{j}(\beta, w)}$. See Section 1.2 of [13] for further details. In this paper, we are interested in the special case $M=\mathbb{C} P^{1}, L=\mathcal{O}(1)$. In order to study the $N^{-1 / 2}$-length scale behavior of $\widehat{\Pi}_{N}$ near a point $\xi \in \mathbb{C} P^{1}$, we recall two definitions from Section 2.2 of [15].

Definition 2. Fix $\xi \in \mathbb{C} P^{1}$ and e a frame for $\mathcal{O}(1)$ in a neighborhood $U$ containing $\xi$. The frame $e$ is called a preffered frame for $h$ at $\xi$ if

$$
\|e(\xi)\|_{h}=1 \text { and } \nabla^{h} e(\xi)=0,
$$

where $\nabla^{h}$ is the metric connection of $h$.

Definition 3. Fix $\xi \in \mathbb{C} P^{1}$, a Kähler normal coordinate $\psi: U \rightarrow \mathbb{C}$ centered at $\xi$, and a preferred frame e for $h$ at $\xi$. A Heisenberg coordinate on $X$ centered at $\xi$ is a coordinate $\rho: S^{1} \times \mathbb{C} \rightarrow \pi^{-1}(U)$ given by

$$
\rho(\theta, \psi(z))=e^{i \theta}\|e(z)\|_{h} e^{*}(z) .
$$

Recall from Sections 2.2 and 2.4 that Hermitian Gaussian ensembles have as a universal scaling limit the Bargman-Fock ensemble in $N^{-1 / 2}$-scale normal coordinates around any point $\xi$. Similarly, when these ensembles are lifted to functions on $X$, they have a universal scaling limit in Heisenberg coordinates. We refer the interested reader to Section 1.3.2 of [3] for more details.

3.4. Szëgo and Bergman Kernel Asymptotics. We now recall for the particular case of $\mathcal{O}(1) \rightarrow \mathbb{C} P^{1}$ the on-diagonal, near off-diagonal, and far off-diagonal asymptotics for the Szëgo kernels $\Pi_{N}$ derived in [13] and [15] by Shiffman and Zelditch. We need them to prove Theorems 1 - 4 .

Theorem 5 (Theorem 1 in [17]). There exists a $C^{\infty}$ complete asymptotic expansion:

$$
\widehat{\Pi}_{N}(\alpha, z ; \beta, z)=\frac{N}{\pi}\left(1+a_{1}(z) \cdot N^{-1}+\ldots\right) \cdot e^{i N(\alpha-\beta)}
$$

for certain smooth coefficients $a_{j}(z)$.

Next, we record a special case of Theorem 2.4 from [15].

Theorem 6. In Heisenberg coordinates on $X$ around $\xi \in \mathbb{C} P^{1}$, for $b>\sqrt{j+2 k}, j, k \geq 0$, we have the following $C^{\infty}$ asymptotic expansions:

1. Far Off-Diagonal. For $d(z, w)>b\left(\frac{\log N}{N}\right)^{1 / 2}$ and $j \geq 0$, we have

$$
\nabla^{j} \widehat{\Pi}_{N}(\alpha, z ; \beta, w)=O\left(N^{-k}\right)
$$


where $\nabla^{j}$ denotes the horizontal lift to $X$ of any $j$ mixed derivatives in $z, \bar{z}, w, \bar{w}$.

2. Near Off-Diagonal. Let $\epsilon>0$. In Heisenberg coordinates (see Definition 3) centered at $\xi$, we have for $|z|+|w|<b\left(\frac{\log N}{N}\right)^{1 / 2}$

$$
\widehat{\Pi}_{N}(\alpha, z ; \beta, w)=e^{i N(\alpha-\beta)-z \cdot \bar{w}+\frac{1}{2}\left(|z|^{2}+|w|^{2}\right)}\left[1+R_{N}(z, w)\right]
$$

where

$$
R_{N}(z, w)=O\left(N^{-1 / 2+\epsilon}\right),
$$

and the implied constant in equation (3.10) is allowed to depend on $\epsilon$.

Finally, we will need to recall the $C^{\infty}$ asymptotic expansions for $P_{N}$.

Theorem 7 (Prop 2.6 and 2.7 from [15]). Let $p_{N}$ be a Gaussian random polynomial defined in Section 1.1, and consider the $N^{\text {th }}$ normalized Szëgo Kernel

$$
P_{N}(z, w)=\frac{\left\|\Pi_{N}(z, w)\right\|_{h^{N}}}{\sqrt{\left\|\Pi_{N}(z, z)\right\|_{h^{N}}\left\|\Pi_{N}(w, w)\right\|_{h^{N}}}} .
$$

We have the following

1. Far Off-Diagonal. For $b>\sqrt{j+2 k}, j, k \geq 0$ and all $|z|+|w|>b\left(\frac{\log N}{N}\right)^{1 / 2}$ we have that

$$
\nabla^{j} P_{N}(z, w)=O\left(N^{-k}\right)
$$

2. Near Off-Diagonal. Let $\epsilon, b>0$ and $\xi \in \mathbb{C} P^{1}$. In Heisenberg coordinates centered at $\xi$ we have

$$
P_{N}(z, w)=e^{-\frac{1}{2}|z-w|^{2}}\left[1+R_{N}(z, w)\right], \quad\left|\nabla^{j} R_{N}(z, w)\right|=O\left(N^{-1 / 2+\epsilon}\right)
$$

where $\nabla^{j}$ denotes any $j$ interated derivatives in $z, \bar{z}, w$, or $\bar{w}$ and the implied constant is uniform in $z, w$ for $|z|+|w|<b\left(\frac{\log N}{N}\right)^{1 / 2}$ and does not depend on $\xi$. The remainder $R_{N}$ satisfies in addition

$$
\left|R_{N}(z, w)\right| \leq C|z-w|^{2} N^{-1 / 2+\epsilon}, \quad\left|\nabla R_{N}(z, w)\right| \leq \frac{C}{2}|z-w| N^{-1 / 2+\epsilon}
$$

for some constant $C$ uniformly for $|z|+|w|<\left(\frac{\log N}{N}\right)^{1 / 2}$.

\section{The Holomorphic Derivative $\frac{d}{d z}$ as a Meromorphic Connection on $\mathcal{O}(N)$}

We use this section to study the meromorphic connection $\nabla^{z_{0}}$ on $\mathcal{O}(N) \rightarrow \mathbb{C} P^{1}$, defined in 1.2 , that extends the euclidean derivative $\frac{d}{d z}$. We give a formal definition in Section 4.1. We then compute various lifts of $\nabla^{z_{0}}$ to the principle $S^{1}$ bundle $X \rightarrow \mathbb{C} P^{1}$ associated to $(\mathcal{O}(1), h)$ in Section 4.2 . These lifts will allow us to obtain asymptotics expansions for covariant derivatives of the Szëgo kernels $\Pi_{N}$ lifted to $X$ in Section 5 . 
4.1. Definition. Let $\mathcal{P}_{N}$ be the space of polynomials of degree at most $N$ in one complex variable. We identify $\mathcal{P}_{N}$ with the space of holomorphic sections $H_{\text {hol }}^{0}\left(\mathbb{C} P^{1}, \mathcal{O}(N)\right)$ by pulling back along the trivialization

$$
\alpha_{N}:\left.\mathcal{O}(N)\right|_{\mathbb{C} P^{1} \backslash\{\infty\}} \stackrel{\cong}{\longrightarrow} \mathbb{C} \times \mathbb{C}
$$

corresponding to the frame $z_{0}^{N}$ (cf Section 2.3). The holomorphic critical points for a holomorphic function $f$ are the zeros of the $(1,0)$-form $d f=\frac{\partial f}{\partial z} d z$. Interpreting $d$ as the trivial connection on $\mathbb{C} \times \mathbb{C}$, we define the meromorphic connection

$$
\nabla^{z_{0}, N}:=\alpha_{N}^{*} d
$$

on $\mathcal{O}(N)$. It is characterized by declaring the section $z_{0}^{N}$ to be parallel. The holomorphic critical points of a degree $N$ polynomial $p_{N}$ are therefore the same as the zeros of

$$
\nabla^{z_{0}, N}\left(p_{N} \cdot z_{0}^{N}\right) \in H_{h o l}^{0}(\mathcal{O}(N)) \otimes \Omega_{m e r}^{(1,0)}\left(\mathbb{C} P^{1}\right) .
$$

We abbreivate $\nabla^{z_{0}}=\nabla^{z_{0}, N}$ throughout and observe that $\nabla^{z_{0}}$ has a pole of order 1 at infinity and is holomorphic otherwise. Indeed, writing $z=\frac{z_{1}}{z_{0}}$ and $w=z^{-1}$ for the standard coordinates around 0 and $\infty$ on $\mathbb{C} P^{1}$, we see that

$$
\nabla^{z_{0}} z_{1}^{N}=\nabla^{z_{0}} z^{N} \cdot z_{0}^{N}=N \cdot z^{N-1} z_{0}^{N} \otimes d z=-\frac{N}{w} \cdot z_{1}^{N} \otimes d w
$$

Since the probability that a guassian random polynomial $p_{N}$ vanishes at infinity is $0, \nabla^{z_{0}} p_{N}$ has a simple pole at infinity almost surely.

4.2. Lift of $\nabla^{z_{0}}$ to Principle $S^{1}$ Bundle. In this section, we compute various lifts of $\nabla^{z_{0}}$ to the principle $S^{1}$ bundle $X \rightarrow \mathbb{C} P^{1}$ associated to a fixed smooth Hermitian metric $h$ on $\mathcal{O}(1)$ (cf Section 3.3). We will denote by $\widehat{\nabla}^{z_{0}}$ the lift of $\nabla^{z_{0}}$ to $X$ and by $\widehat{\nabla}^{z_{0}}(1,0)$ and $\widehat{\nabla}^{z_{0}}(0,1)$ its $(1,0)$ and $(0,1)$ parts.

We continue to write $\phi_{z_{0}}: \mathbb{C} P^{1} \backslash\{\infty\} \rightarrow \mathbb{R}$ for the Kähler potential

$$
\phi_{z_{0}}(z)=\log \left\|z_{0}(z)\right\|_{h}^{-2}
$$

over $\mathbb{C} P^{1} \backslash\{\infty\}$ for $\omega_{h}$. For $\xi \in \mathbb{C} P^{1} \backslash\{\infty\}$ and any holomorphic coordinate $z$ centered at $\xi$, we write $\gamma_{0}$ for the "leading harmonic part" of $\phi_{z_{0}}$ :

$$
\gamma_{0}(z, \bar{z}):=\phi_{z_{0}}(\xi)+\frac{\partial \phi_{z_{0}}}{\partial z}(\xi) \cdot z+\frac{\partial \phi_{z_{0}}}{\partial \bar{z}}(\xi) \cdot \bar{z}+\frac{1}{2}\left[\frac{\partial^{2} \phi_{z_{0}}}{\partial z^{2}} \cdot z^{2}+\frac{\partial^{2} \phi_{z_{0}}}{\partial \bar{z}^{2}} \cdot \bar{z}^{2}\right] .
$$

Similarly, writing as in Section $2.3 z_{1}$ for the standard frame of $\mathcal{O}(1)$ over $\mathbb{C} P^{1} \backslash\{0\}$, we define $\phi_{z_{1}}:=\log \left\|z_{1}\right\|_{h}^{-2}$ and introduce

$$
\gamma_{1}(z, \bar{z}):=\phi_{z_{1}}(\xi)+\frac{\partial \phi_{z_{1}}}{\partial z}(\xi) \cdot z+\frac{\partial \phi_{z_{1}}}{\partial \bar{z}}(\xi) \cdot \bar{z}+\frac{1}{2}\left[\frac{\partial^{2} \phi_{z_{1}}}{\partial z^{2}} \cdot z^{2}+\frac{\partial^{2} \phi_{z_{1}}}{\partial \bar{z}^{2}} \cdot \bar{z}^{2}\right]
$$

Lemma 1 (Lift of $\nabla^{z_{0}}$ in Heisenberg Coordinates). Fix $\xi \in \mathbb{C} P^{1}$ and a Heisenberg coordinate on $X$ centered at $\xi$. If $\xi \neq \infty$, then we may write

$$
\widehat{\nabla}^{z_{0}}(1,0)(\alpha, z)=\partial_{z}+\frac{N}{2} \cdot \partial_{z}\left[\phi_{z_{0}}(z)+\gamma_{0}(z)\right] .
$$

Further, fix Heisenberg coordinates centered at $\xi$ on the diagonal of $X \times X$. The (differential) order 0 part of the lift of $\nabla^{z_{0}} \otimes \overline{\nabla^{z_{0}}}$ is

$$
N \partial_{z} \bar{\partial}_{z} \phi_{z_{0}}(z)+N^{2} \partial_{z} \phi_{z_{0}} \cdot \bar{\partial}_{z} \phi_{z_{0}}(z) \text {. }
$$


The order 1 and 2 parts are $O(N)$. Finally, if $\xi=\infty$ and we write $\psi(z)=w$ for the change of coordinates to the usual holomorphic coordinate $w=\frac{z_{0}}{z_{1}}$ at $\xi$, we have

$$
\widehat{\nabla}^{(1,0)}(\alpha, z)=\partial_{z}+\frac{N}{2} \cdot \partial_{z}\left[\phi_{z_{1}}(z)+\gamma_{1}(z)+\log \psi(z)^{2}\right] .
$$

In Heisenberg coordinates centered at $\infty$ on the diagonal of $X \times X$, the lift of the (differential) order 0 part of $\nabla^{z_{0}} \otimes \overline{\nabla^{z_{0}}}$ is

$$
\frac{N^{2}}{|\psi(z)|^{2}}+N \operatorname{Re}\left(\frac{\partial_{z} \phi_{z_{1}}(\psi(z))}{\psi(z)}\right)+N \partial_{z} \bar{\partial}_{z} \phi_{z_{0}}(\psi(z))+N^{2} \partial_{z} \phi_{z_{0}}(\psi(z)) \cdot \bar{\partial}_{z} \phi_{z_{0}}(\psi(z)) \text {. }
$$

The order 1 and 2 parts are $O(N)$.

Taylor expanding the results of Lemma 1, gives the following corollary.

Corollary 1 (Lift of $\nabla^{z_{0}}$ in Scaled Heisenberg Coordinates). Fix $\xi$ and a scaled Heisenberg coordinate centered at $\xi$. In the notation of Lemma 1 , the lifted connection $\widehat{\nabla^{z_{0}}}$ exhibits three different behaviors.

Case $1 d \phi_{z_{0}}(\xi) \neq 0$. If $d \phi_{z_{0}(\xi)} \neq 0$ or $\xi \neq \infty$, then we have

$$
\widehat{\nabla}^{\left(z_{0}\right.}(1,0)(u, \theta)=\left.\frac{\partial \phi_{z_{0}}}{\partial u}\right|_{\xi} \otimes N^{1 / 2} \cdot\left(1+O\left(N^{-1 / 2}\right)\right) d u
$$

and the (differential) order 0 part of the $(1,1)$ part of $\nabla^{z_{0}} \otimes \overline{\nabla^{z_{0}}}$ lifted to the diagonal is

$$
\left.\left|\frac{\partial \phi_{z_{0}}}{\partial u}\right|_{\xi}\right|^{2} \otimes N \cdot\left(1+O\left(N^{-1 / 2}\right)\right) \cdot d u \wedge d \bar{u} \text {. }
$$

Case $2 d \phi_{z_{0}}(\xi)=0$. If $d \phi_{z_{0}}(\xi)=0$ and $\xi \neq \infty$ then

$$
{\widehat{\nabla^{z}}}^{(1,0)}(u, \theta)=\partial_{u}+\frac{1}{2}\left(\left.\frac{\partial^{2} \phi_{z_{0}}}{\partial u^{2}}\right|_{\xi} \cdot u+\bar{u}\right) \otimes d u+O\left(N^{-1 / 2}\right),
$$

and the (differential) order 0 part of the $(1,1)$ part of $\nabla^{z_{0}} \otimes \overline{\nabla^{z_{0}}}$ lifted to the diagonal is

$$
\left(1+\left|\frac{\partial^{2} \phi_{z_{0}}}{\partial u^{2}}\right|_{\xi} \cdot u+\left.\bar{u}\right|^{2}+O\left(N^{-1 / 2}\right)\right) \otimes d u \wedge d \bar{u}
$$

Case $3 \xi=\infty$. Finally, in the case $\xi=\infty$, we have

$$
{\widehat{\nabla^{z_{0}}}}^{(1,0)}(u, \theta)=-\left.\frac{\partial \psi}{\partial w}\right|_{\xi} \cdot \frac{1}{u} \otimes N\left(1+O\left(N^{-1 / 2}\right)\right) d u,
$$

and the (differential) order 0 part of the $(1,1)$ part of $\nabla^{z_{0}} \otimes \overline{\nabla^{z_{0}}}$ lifted to the diagonal is

$$
|u|^{-2} \otimes N^{3}\left(1+O\left(N^{-1 / 2}\right)\right) \cdot d u \wedge d \bar{u} .
$$

Remark 6. Equation (4.6) shows that in scaled Heisenberg coordinates centered at $\xi$ satisfying $d \phi_{z_{0}}(\xi) \neq 0$ or $\xi=\infty, \widehat{\nabla^{z_{0}}}$ is an order 0 operator to leading order in $N$. This explains analytically why, in the large $N$ limit, zeros and critical points are indistinguishable in this case. In contrast, (4.8) shows that $\widehat{\nabla^{z_{0}}}$ is an order 1 operator to leading order in $N$ if $d \phi_{z_{0}}(\xi)=0$. 
Proof of Lemma 1. First suppose that $\xi \in \mathbb{C} P^{1} \backslash\{\infty\}$. We begin by constructing Heisenberg coordinates on $X$ centered at $\xi$. With $\widetilde{\gamma}_{0}$ denoting the harmonic conjugate of $\gamma_{0}$, we observe that the frame

$$
e_{L}:=e^{\frac{1}{2}\left(\gamma_{0}+i \widetilde{\gamma}_{0}\right)} \cdot z_{0}
$$

is a preffered frame near $\xi$ in the sense of Definition 2. Combined with any Kähler normal coordinate centered at $\xi$, the frame $e_{L}$ allows us to construct Heisenberg coordinates centered at $\xi$. Note that

$$
\left\|e_{L}\right\|_{h}^{N}=e^{\frac{N}{2}\left(\gamma_{0}-\phi_{z_{0}}\right)} .
$$

Fix $S \in H_{\text {hol }}^{0}\left(\mathbb{C} P^{1}, \mathcal{O}(N)\right)$, and write $S=f \cdot e_{L}$ locally. Since

$$
\frac{\partial \gamma_{0}}{\partial z}=i \frac{\partial \widetilde{\gamma_{0}}}{\partial z}
$$

we use expression (3.4) for lifting sections of $\mathcal{O}(N)$ to functions on $X$ to write

$$
\widehat{\nabla_{\frac{\partial}{\partial z}}^{z_{0}} S}(\alpha, z)=\left(\frac{\partial f}{\partial z}(z)+N f(z) \frac{\partial \gamma_{0}}{\partial z}(z)\right) e^{\frac{N}{2}\left(\gamma_{0}(z)-\phi_{z_{0}}(z)\right)} e^{i N \theta} .
$$

Therefore, the $(1,0)$ part of the lift of the connection $\nabla^{z_{0}}$ on $\mathcal{O}(N)$ to the trivial line bundle $X \times \mathbb{C}$ is

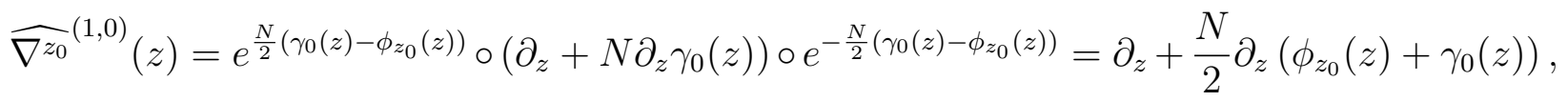

where $\circ$ denotes composition of differential operators. This confirms 4.2). Next, to deduce 4.3, we use the definition of Heisenberg coordinates (3.6) to write

$$
\widehat{S \otimes \bar{S}}(\alpha, z ; \beta, z)=|f(z)|^{2} e^{N\left(\gamma(z)-\phi_{z_{0}}(z)\right)} e^{i N(\alpha-\beta)} .
$$

Similarly, the lift of $\nabla_{\frac{\partial}{\partial z}}^{z_{0}} S(z) \otimes \bar{\nabla}_{\frac{\partial}{\partial z}}^{z_{0}} S(z)$ to $X \times X$ :

$$
\left|\frac{\partial f}{\partial z}(z)+N f \frac{\partial \gamma}{\partial z}(z)\right|^{2} e^{N\left(\gamma(z)-\phi_{z_{0}}(z)\right)} e^{i N(\alpha-\beta)} .
$$

Comparing the two previous expressions, we see that the $(1,1)$ part of the lift of $\nabla^{z_{0}} \otimes \overline{\nabla^{z_{0}}}$ to the diagonal of $X \times X$ is given by

$$
\left.\left[e^{N\left(\gamma_{0}(z, w)-\phi_{z_{0}}(z, w)\right)} \circ\left(\partial_{z}+N \partial_{z} \gamma_{0}(z)\right) \circ\left(\bar{\partial}_{w}+N \bar{\partial}_{w} \gamma_{0}(w)\right) \circ e^{-N\left(\gamma_{0}(z, w)-\phi_{z_{0}}(z, w)\right)}\right]\right|_{z=w} .
$$

Here $\gamma(z, w)$ and $\phi_{z_{0}}(z, w)$ denote the extensions of $\gamma$ and $\phi_{z_{0}}$ from the diagonal of $X \times X$ that are holomorphic in $z$ and anti-holomorphic in $w$. The expression (4.13) may be written as

$$
\underbrace{N \partial_{z} \bar{\partial}_{z} \phi_{z_{0}}+N^{2} \partial_{z} \phi_{z_{0}} \cdot \bar{\partial}_{z} \phi_{z_{0}}}_{\text {order } 0}+\underbrace{N \partial_{z} \phi_{z_{0}} \bar{\partial}_{z}+N \bar{\partial}_{z} \phi_{z_{0}} \partial_{z}}_{\text {order } 1}+\underbrace{\partial_{z} \bar{\partial}_{z}}_{\text {order } 2}
$$

confirming 4.3.

Finally, we consider the case when $\xi=\infty$. This case needs to be treated separately since no parallel frame for $\nabla^{z_{0}}$ exists near infinity. We write $z_{1}$ for the usual frame of $\mathcal{O}(1)$ over $\mathbb{C} P^{-}\{0\}$ (cf Section 2.3). As before,

$$
e_{L}:=e^{\frac{1}{2}\left(\gamma_{1}+i \widetilde{\gamma}_{1}\right)} z_{1}
$$


is a preferred frame for $\mathcal{O}(1)$ near $\xi=\infty$. Recall from (4.1) that if we denote by $w$ the standard coordinate around $\infty$,

$$
\nabla^{z_{0}} z_{1}^{N}=-\frac{N}{w} z_{1}^{N} \otimes d w
$$

Write $z=\psi(w)$ for the change of coordinates to a Kähler normal coordinate for $\omega_{h}$ centered at $\xi$. Note that $\frac{\partial \gamma_{1}}{\partial w}=i \frac{\partial \widetilde{\gamma}_{1}}{\partial w}$. For $S=f \cdot e_{L} \in H_{h o l}^{0}\left(\mathbb{C} P^{1}, \mathcal{O}(N)\right)$ as before

$$
\widehat{\nabla_{\frac{\partial}{\partial z}}^{z_{0}} S}(\theta, z)=\left(\frac{\partial f}{\partial z}(z)+N f(z)\left[\frac{\partial \gamma_{1}}{\partial z}(z)+\frac{1}{\psi(z)} \frac{\partial \psi}{\partial z}(z)\right]\right) e^{\frac{N}{2}\left(\gamma_{1}(z)-\phi_{z_{1}}(z)\right)} e^{i N \theta} .
$$

The $(1,0)$ part of the lift of the connection $\nabla^{z_{0}}$ on $\mathcal{O}(N)$ to the trivial line bundle $X \times \mathbb{C}$ is therefore

$$
\widehat{\nabla}^{(1,0)}(\alpha, z)=\partial_{z}+\frac{N}{2} \cdot \partial_{z}\left[\phi_{z_{1}}(z)+\gamma_{1}(z)+\log \psi(z)^{2}\right]
$$

confirming 4.4. Equation (4.5) is derived exactly like 4.3).

\section{Asymptotics of $\nabla^{z_{0}}$ Derivatives of the Szego Kernel}

We now combine the formulas from Section 4.2 for the different lifts of $\nabla^{z_{0}}$ to $X$ with the asymptotics of Theorems 6 and 7 to derive asymptotic expasions for the covariant derivatives of $\widehat{\Pi}_{N}$ and $P_{N}$ with respect to $\nabla^{z_{0}}$.

Theorem 8. There exists a $C^{\infty}$ complete asymptotic expansion for lift of $\nabla^{z_{0}} \otimes \overline{\nabla^{z_{0}}} \Pi_{N}$ to the diagonal in $X \times X$ :

$$
\left[N \frac{\partial^{2} \phi_{z_{0}}}{\partial z \partial \bar{z}}+N^{2}\left|\frac{\partial \phi_{z_{0}}}{\partial z}(z)\right|^{2}+O(1)\right] \otimes d z \wedge d \bar{z}
$$

Proof. Equation (5.1) follows immediately from the asymptotic expansion (5.1) of Theorem 5 and the lift of $\nabla^{z_{0}} \otimes \overline{\nabla^{z_{0}}}$ to the diagonal of $X \times X$ given in 4.3 of Lemma 1 .

We now use the asymptotic expansions for $\widehat{\Pi}_{N}(z, w)$ given in Theorem 6 combined with Lemma 1 and Corollary 1 to control $\widetilde{P}_{N}(z, w)$, the correlation between $p_{N}(z)$ and $\nabla^{z_{0}} p_{N}(w)$.

Theorem 9. We use the notation of Theorem 6 and consider

$$
\widetilde{P}_{N}(z, w)=\frac{\left\|1 \otimes \overline{\nabla_{V}^{z_{0}}} \Pi_{N}(z, w)\right\|_{h^{N}}}{\left[\left\|\Pi_{N}(z, z)\right\|_{h^{N}}\left\|\nabla_{V}^{z_{0}} \otimes \overline{\nabla_{V}^{z_{0}}} \Pi_{N}(w, w)\right\|_{h^{N}}\right]^{1 / 2}} .
$$

1. Far Off-Diagonal Asymptotics. For $b>\sqrt{j+2 k}, j, k \geq 0$, we have

$$
\nabla^{j} \widetilde{P}_{N}(z, w)=O\left(N^{-k}\right)
$$

uniformly for $|z-w| \geq b \cdot\left(\frac{\log N}{N}\right)^{1 / 2}$.

2. Near Off-Diagonal Asymptotics. Let $\epsilon>0$ and $\xi \in \mathbb{C} P^{1}$. Take a $N^{-1 / 2}$-scale normal coordinate centered at $\xi$ and write $\phi_{z_{0}}:=\log \left\|z_{0}\right\|_{h}^{-2}$. If $d \phi_{z_{0}}(\xi)=0$, then

$$
\widetilde{P}_{N}(z, w)=\frac{\left|\frac{\partial^{2} \phi_{z_{0}}}{\partial \bar{w}^{2}}\right|_{\xi} \cdot \bar{w}+z \mid}{\sqrt{1+\left|\frac{\partial^{2} \phi_{z_{0}}}{\partial \bar{w}^{2}}\right|_{\xi} \cdot \bar{w}+\left.w\right|^{2}}} e^{-\frac{1}{2}|z-w|^{2}}\left[1+\widetilde{R}_{N}(z, w)\right] .
$$


If $d \phi_{z_{0}}(\xi) \neq 0$ or $\xi=\infty$, then

$$
\widetilde{P}_{N}(z, w)=e^{-\frac{1}{2}|z-w|^{2}}\left[1+\widetilde{R}_{N}(z, w)\right] .
$$

The remainders $\widetilde{R}_{N}$ in (5.3) and (5.4) satisfy $\left|\nabla^{j} \widetilde{R}_{N}(z, w)\right|=O\left(N^{-1 / 2+\epsilon}\right)$ as well as

$$
\left|\widetilde{R}_{N}(z, w)\right| \leq C|z-w| N^{-1 / 2+\epsilon}
$$

for some constant $C$ uniformly for $|z|+|w|<\left(\frac{\log N}{N}\right)^{1 / 2}$.

Proof. Equations (5.2) and (5.3) follow immediately by differentiating $(5.2)$ and (5.3) by the lift of $\nabla^{z_{0}}$ to $X$ given in 4.6 and 4.8 .

We conclude by recording some estimates on $\widetilde{P}_{N}$ that we will be useful in proving Theorems 1 and 2 .

Corollary 2. Fix $\xi \in \mathbb{C} P^{1}$ and a $N^{-1 / 2}$-scale Kähler normal coordinate centered at $\xi$. If $d \phi_{z_{0}}(\xi)=0$, then

$$
\widetilde{P}_{N}(z, w) \leq C<1
$$

uniformly in $N$ for some universal constant $C$ for all $|z-w| \leq \log N$. If $d \phi_{z_{0}}(\xi) \neq 0$ or $\xi=\infty$, then

$$
\widetilde{P}_{N}(z, w) \leq 1-K \cdot \frac{1}{\sqrt{N}}
$$

uniformly in $N$ for some constant $K>0$ and for all $|z-w| \leq \log N$. If we also assume that $|z-w|=O\left(N^{-1 / 4}\right)$, then for a constant $R>0$

$$
\begin{aligned}
P_{N}^{\xi}(z, w)^{2} & =1-|z-w|^{2}-R \cdot N^{-1 / 2}+O\left(N^{-3 / 4+\epsilon}\right) \\
\partial_{z}\left(P_{N}^{\xi}(z, w)^{2}\right) & =O\left(N^{-1 / 4}\right) \\
\partial_{z} \bar{\partial}_{z} P_{N}^{\xi}(z, w)^{2} & =1+O\left(N^{-1 / 2+\epsilon}\right) .
\end{aligned}
$$

Proof. Fix $\xi \in \mathbb{C} P^{1}$ and a $N^{-1 / 2}$-scale Kähler normal coordinate centered at $\xi$. Suppose first that $d \phi_{z_{0}}(\xi)=0$. Equation (5.3) implies

$$
\widetilde{P}_{N}(z, w)=\frac{|\alpha \bar{w}+z|}{\sqrt{1+|\alpha \cdot \bar{w}+w|^{2}+O\left(N^{-1}\right)}} \cdot e^{-\frac{1}{2}|z-w|^{2}}\left(1+O\left(N^{-1 / 2}\right)\right),
$$

where we've denoted $\alpha=\frac{\partial^{2} \phi_{z_{0}}}{\partial \bar{w}^{2}}(\xi)$. Setting $\eta=\alpha \bar{w}+w$ and $\xi=z-w$, we have

$$
\widetilde{P}_{N}^{2}(z, w)=\frac{|\eta+\xi|^{2}}{1+|\eta|^{2}} e^{-|\xi|^{2}}
$$

When $|\xi|$ is large, 5.6 is satisfied. For $|\xi|$ bounded above, we taylor expand $e^{-|\xi|^{2}}$ to write

$$
\widetilde{P}_{N}^{2}(z, w) \leq \frac{|\eta+\xi|^{2}}{\left(1+|\eta|^{2}\right)\left(1+|\xi|^{2}+|\xi|^{4} / 2\right)}+O\left(N^{-1 / 2}\right)
$$

Estimating the numerator above by $|\xi|^{2}+2|\xi||\eta|+|\eta|^{2}$ and using that $1+|\xi|^{2}|\eta|^{2} \geq 2|\xi||\eta|$ confirms (5.6). 
Suppose next that $d \phi_{z_{0}}(\xi) \neq 0$ or $\xi=\infty$. The asymptotic expansion $(5.2)$ yields

$$
\widetilde{P}_{N}(z, w)=\frac{\beta}{\sqrt{\beta^{2}+O\left(N^{-1 / 2}\right)}} \cdot e^{-\frac{1}{2}|z-w|^{2}}\left(1+O\left(N^{-1 / 2}\right)\right),
$$

where we set $\beta:=\left|\frac{\partial \phi_{z_{0}}}{\partial z}(\xi)\right|$. Estimating $e^{-\frac{1}{2}|z-w|^{2}} \leq 1$ we see that

$$
P_{N}(z, w) \leq 1-\frac{K}{\sqrt{N}}
$$

for some $K>0$, as desired. Finally, assume additionally that $|z-w|=O\left(N^{-1 / 4}\right)$. Writing $e^{-|z-w|^{2}}=1-|z-w|^{2}+O\left(N^{-1}\right)$ in $(5.11)$, we conclude (5.8). Equations (5.9) and (5.10) now easily follow from (5.8) and the remainder estimate (5.5).

\section{Relation of Szëgo Kernels to Zeros and Critical Points}

In this section, we give explicit formulas for $E\left[Z_{p_{N}}\right]$ and $E\left[C_{p_{N}}\right]$ in terms of the Szëgo Kernels $\Pi_{N}$ and the connection $\nabla^{z_{0}}$. Lemma 2 is a rather general and simple result that was proved in various guises in [1, 6, 14] and essentially in the present form as Proposition 2.1 in [15]. Both its conclusion and the ideas in its proof will be used throughout.

Lemma 2 (Probabilistic Poincare-Lelong Formula). Let $M$ be a complex manifold without boundary and $L \rightarrow M$ be a holomorphic line bundle endowed with a positive Hermitian metric h. Let

$$
\left\{\Sigma_{j}, j=1, \ldots, J\right\} \subset H_{m e r}^{0}(M, L)
$$

be arbitrary merormorphic sections that are not all identically zero. Define a Gaussian random section $s$ by

$$
s(z):=\sum_{j=1}^{J} a_{j} \Sigma_{j}(z), a_{j} \sim N(0,1)_{\mathbb{C}} \text { i.i.d. }
$$

Denoting by $E[\cdot]$ the expected value operator for the standard complex Gaussian vector $\left[a_{1}, \ldots, a_{J}\right]$ and by $Z_{s}$ and $P_{s}$ the currents of integration over the zeros and poles of $s$, we have

$$
E\left[Z_{s}-P_{s}\right](z)=\frac{i}{2 \pi} \partial \bar{\partial} \log \|\Pi(z, z)\|_{h}+\omega_{h}(z)
$$

Here $\omega_{h}$ is first chern class of $(L, h)$ and $\Pi(z, w):=\sum_{j=1}^{N} \Sigma_{j}(z) \otimes \overline{\Sigma_{j}(w)}$ is the associated Szëgo kernel. In a local holomorphic frame $e_{L}$ of $L$, we write $\Sigma_{j}=\sigma_{j} \cdot e_{L}$ and obtain the following equivalent expression:

$$
E\left[Z_{s}-P_{s}\right](z)=\frac{i}{2 \pi} \partial \bar{\partial} \log \sum_{j=1}^{J}\left|\sigma_{j}(z)\right|^{2} .
$$

Lemma 2 is a probabilitist analog of the following well-known result:

Lemma 3 (Poincare-Lelong Formula). Let $L \rightarrow M$ be a holmorphic line bundle over a complex manifold and suppose $s \in H_{\text {hol }}^{0}(M, L)$ is a merormophic section. Write $Z_{s}$ and $P_{s}$ for the currents of integration over the zeros and poles of $s$ and express $s=f \cdot e$ relative to a local frame e of $L$. Then

$$
Z_{s}-P_{s}=\frac{i}{\pi} \partial \bar{\partial} \log |f| .
$$


Proof of Lemma 2. To prove (6.1), it is enough to verify (6.2) as for any local frame $e_{L}$ of $L, \omega_{h}$ is given locally by $\frac{i}{2 \pi} \partial \bar{\partial} \log \left\|e_{L}\right\|_{h}^{-2}$, making

$$
\frac{i}{2 \pi} \partial \bar{\partial} \log \|\Pi(z, z)\| \cdot\left\|e_{L}\right\|_{h}^{-2}=\frac{i}{2 \pi} \partial \bar{\partial} \log \sum_{j=1}^{J}\left|\sigma_{j}(z)\right|^{2}
$$

the local expression for

$$
\frac{i}{2 \pi} \partial \bar{\partial} \log \left\|\Pi_{N}(z, z)\right\|_{h}+\omega_{h}(z) .
$$

We will abbreviate $s=\langle a, \Sigma\rangle=\langle a, \sigma\rangle \cdot e_{L}$, where

$$
a=\left[a_{0}, \ldots, a_{J}\right], \Sigma=\left[\Sigma_{0}, \ldots, \Sigma_{J}\right], \sigma=\left[\sigma_{0}, \ldots, \sigma_{J}\right] .
$$

For any smooth test function $\psi$, we apply the Poincare-Lelong formula to write

$$
\begin{aligned}
\left(E\left[Z_{s}-P_{s}\right], \psi\right) & =\frac{i}{\pi} E[(\log |\langle a, \sigma\rangle|, \partial \bar{\partial} \psi)] \\
& =\frac{i}{2 \pi}\left(\log \sum_{j=0}^{N}\left|\sigma_{j}\right|^{2}, \partial \bar{\partial} \psi\right)+\frac{i}{\pi} E[(\log |\langle a, u\rangle|, \partial \bar{\partial} \psi)] .
\end{aligned}
$$

We've set

$$
u(z)=\frac{\sigma(z)}{\sum_{j=0}^{N}\left|\sigma_{j}\right|^{2}},
$$

a unit vector at all but finitely many points. The second term vanishes due to the unitary invariance of guassian measure. Indeed, as in Section 3.2 of [12], we write the second term as

$$
\frac{i}{\pi} \int_{\mathbb{C}^{N+1}}\left[\int_{\mathbb{C} P^{1}} \log |\langle a, u(z)\rangle| \partial_{z} \bar{\partial}_{z} \psi(w) d z d \bar{z}\right] d \gamma(a),
$$

where $d \gamma(a)=\frac{1}{\pi^{J+1}} e^{-\|a\|^{2}} d a$ is the Gaussian density on $\mathbb{C}^{J+1}$. It is straight-forward to check that the integrand is in $L^{1}$, allowing us to change the order of integration. For almost every $z \in \mathbb{C} P^{1}$, we have that $\langle a, u(z)\rangle \stackrel{\mathcal{D}}{=} a_{1}$, a standard normal random variable on $\mathbb{C}$. The integral

$$
\int_{\mathbb{C}^{N+1}} \log |\langle a, u(z)\rangle| d \gamma(a)
$$

is therefore a universal constant independent of $z$ and is killed by the operator $\partial_{z} \bar{\partial}_{z}$.

We have the following

Corollary 3. Let $p_{N}$ be a degree $N$ polynomial drawn from the Hermitian Gaussian Ensemble corresponding to a smooth positive Hermitian metric $h$ on $\mathcal{O}(1)$. Let $\omega_{h}$ denote the first chern class of $(\mathcal{O}(1), h)$. Write $C_{p_{N}}$ for the current of integration over the critical point set of $p_{N}$. For any $\xi \in \mathbb{C} P^{1}$ and any non-vanishing holomorphic vector field $V$ in a neighbhorhood of $\xi$, we have

$$
E\left[C_{p_{N}}\right](\xi)=\frac{i}{2 \pi} \partial \bar{\partial} \log \left[\left\|\nabla_{V}^{z_{0}} \otimes \nabla_{\bar{V}}^{z_{0}} \Pi_{N}(\xi, \xi)\right\|_{h}\right]+N \cdot \omega_{h}(\xi)+\delta_{\infty}(\xi) .
$$

Consequently, $E\left[C_{p_{N}}\right]$ is a smooth $(1,1)$-form. 
Proof. By definition, we may write locally

$$
C_{p_{N}}=Z_{\nabla_{V}^{z_{0}} p_{N}}
$$

where $V$ is any non-vanishing holomorphic vector field. Recall from Section 4 that $\nabla_{V}^{z_{0}} p_{N}$ is holomorphic except at $\infty$ where it has a simple pole almost surely. Therefore, denoting by $P_{s}$ the current of integration over the poles for a section $s \in H_{m e r}^{0}\left(\mathbb{C} P^{1}, \mathcal{O}(N)\right)$, we have

$$
E\left[P_{\nabla_{V}^{z_{0}} p_{N}}\right]=\delta_{\infty}
$$

Combining the Poincare-Lelong formula with Lemma 2 applied to $M=\mathbb{C} P^{1}, L=(\mathcal{O}(N), h)$, $\omega=w_{h^{N}}$ and the Gaussian random section $p_{N}$ proves (6.3). That $E\left[C_{p_{N}}\right]$ is a smooth $(1,1)$ - form away from infinity is clear from $(6.3)$. To check that $E\left[C_{p_{N}}\right]$ is smooth at $\infty$ we choose $\left\{S_{j}\right\}_{j=0}^{N}$, an orthonormal basis of $H_{h o l}^{0}\left(\mathbb{C} P^{1}, \mathcal{O}(N)\right)$ with respect to the inner product (1.1) and compute in the standard holomorphic coorinate centered at $\infty$. Relative to the usual frame $z_{1}$ of $\mathcal{O}(1)$ over $\mathbb{C} P^{1} \backslash\{0\}$, we may write $S_{j}=f_{j} \cdot z_{1}^{N}$. By 4.1 ,

$$
\left\|\nabla^{z_{0}} \otimes \overline{\nabla^{z_{0}}} \Pi_{N}(z, w)\right\|_{h^{N}}=N^{2} \frac{\left\|z_{1}\right\|_{h}^{2 N}}{|w|^{2}} \cdot \sum_{j=0}^{N}\left|\frac{\partial f_{j}}{\partial w}(w)\right|^{2} .
$$

Note that $\sum_{j=0}^{N}\left|\frac{\partial f_{j}}{\partial w}(w)\right|^{2}>0$. Writing

$$
\delta_{\infty}(w)=\frac{i}{\pi} \partial_{w} \bar{\partial}_{w} \log |w|^{2} \quad \text { and } \quad N \cdot \omega_{h}(w)=\frac{i}{2 \pi} \partial_{w} \bar{\partial}_{w} \log \left\|z_{1}(w)\right\|_{h}^{-2 N}
$$

in 6.3$)$ shows that $E\left[C_{p_{N}}\right]$ is smooth at infinity.

\section{Expected Density of Critical Points: Proof of Theorem 4}

Throughout, we denote by $p_{N}$ a degree $N$ polynomial drawn from the Hermitian Gaussian Ensemble corresponding to a smooth positive Hermitian metric $h$ on the line bundle $\mathcal{O}(1) \rightarrow$ $\mathbb{C} P^{1}$ (see Section 1.1). We continue to write $\nabla^{z_{0}}$ for both the meromorphic connection on $\mathcal{O}(1)$ that extends the euclidean derivative (see Section 4 ) and the connections $\left(\nabla^{z_{0}}\right)^{\otimes N}$ it induces on $\mathcal{O}(N) \rightarrow \mathbb{C} P^{1}$.

Proof of Theorem 4. Since $E\left[C_{p_{N}}\right]$ is smooth by Corollary 3 , to verify the asymptotics for $\frac{1}{N-1} E\left[C_{p_{N}}\right]$ given in equation 1.15 it suffices to show that for each $\psi \in C_{c}^{2}\left(\mathbb{C} P^{1} \backslash\{\infty\}\right)$ we have

$$
\int_{\mathbb{C} P^{1} \backslash\{\infty\}} \psi(z) E\left[C_{p_{N}}\right](z)=\int_{\mathbb{C} P^{1} \backslash\{\infty\}} \psi(z) \cdot N \omega_{h}(z)+O(1)
$$

with the implied constant independent of $N$. We write $z$ for the standard holomorphic coordinate on $\mathbb{C} P^{1} \backslash\{\infty\}$ and $z_{0}$ for the standard frame of $\mathcal{O}(1)$ over $\mathbb{C} P^{1} \backslash\{\infty\}$ as in Section 2.3. From equation (4.3) and the asymptotic expansion (5.1), we conclude that

$$
\left\|\nabla_{\frac{\partial}{\partial z}}^{z_{0}} \otimes \bar{\nabla}_{\frac{\partial}{\partial z}}^{z_{0}} \Pi_{N}(z, z)\right\|_{h^{N}}=\left.\left|\frac{\partial^{2}}{\partial z \partial \bar{z}} \phi_{z_{0}}(z)+N\right| \frac{\partial \phi_{z_{0}}}{\partial z}(z)\right|^{2}+O\left(N^{-1}\right) \mid .
$$


The assumption that $h$ is a postive metric means that $\frac{\partial^{2}}{\partial z \partial \bar{z}} \phi_{z_{0}}(z)>0$. So we may omit the absolute values in the previous line and use (6.3) of Corollary 3 to write

$$
E\left[C_{p_{N}}\right](z)-N \cdot \omega_{h}(z)=\frac{i}{2 \pi} \partial \bar{\partial} \log \left[\frac{\partial^{2}}{\partial z \partial \bar{z}} \phi_{z_{0}}(z)+N\left|\frac{\partial \phi_{z_{0}}}{\partial z}(z)\right|^{2}\right]+O\left(N^{-1}\right) .
$$

Hence, we seek to show that

$$
\int_{\mathbb{C} P^{1} \backslash\{\infty\}} \frac{i}{2 \pi} \partial \bar{\partial} \log \left[\frac{\partial^{2}}{\partial z \partial \bar{z}} \phi_{z_{0}}(z)+N\left|\frac{\partial \phi_{z_{0}}}{\partial z}(z)\right|^{2}\right] \psi(z)=O(1) .
$$

Note that

$$
\log \left[\frac{\partial^{2}}{\partial z \partial \bar{z}} \phi_{z_{0}}(z)+N\left|\frac{\partial \phi_{z_{0}}}{\partial z}\right|^{2}\right] \geq \log \left(\inf _{\mathbb{C} P^{1}} \frac{\partial^{2}}{\partial z \partial \bar{z}} \phi_{z_{0}}(z)\right)=: c .
$$

Hence, integrating by parts, we have that 7.3 is bounded below by $c \cdot \int_{\mathbb{C} P^{1}} \partial \bar{\partial} \psi$. We also have that

$$
\partial \bar{\partial} \log \left[\frac{\partial^{2}}{\partial z \partial \bar{z}} \phi_{z_{0}}(z)+N\left|\frac{\partial \phi_{z_{0}}}{\partial z}\right|^{2}\right]=\partial \bar{\partial} \log \left[\left|\frac{\partial \phi_{z_{0}}}{\partial z}\right|^{2}+O\left(N^{-1}\right)\right]
$$

Again integrating by parts, we see that $\sqrt{7.3}$ is bounded above by $C \cdot \int \partial \bar{\partial} \psi$, with

$$
C:=\sup _{\operatorname{supp}(\psi)} \log \left|\frac{\partial \phi_{z_{0}}}{\partial z}\right|
$$

This completes the proof of 1.15$)$.

To prove the local asymptotics $(1.17)$ and $(1.16)$, we fix $\xi \in \mathbb{C} P^{1}$ and take a $N^{-1 / 2}$-scale normal coordinate $w$ centered at $\xi$. As noted in (2.6),

$$
N \cdot \omega_{h}(z)=\frac{i}{2 \pi} \partial \bar{\partial}|z|^{2}+O\left(N^{-1 / 2}\right) .
$$

Next, combining the asymptotic expansion (5.1) for $\Pi_{N}(w, w)$ with the lift of $\nabla^{z_{0}} \otimes \nabla^{z_{0}}$ to the diagonal of $X \times X$ given in (4.7), we see that if $d \phi_{z_{0}}(\xi) \neq 0$ or $\xi=\infty$, then

$$
\left\|\nabla_{\frac{\partial}{\partial z}}^{z_{0}} \otimes \bar{\nabla}_{\frac{\partial}{\partial z}}^{z_{0}} \Pi_{N}(w, w)\right\|_{h^{N}}=N \cdot\left(\left.\left|\frac{\partial \phi_{z_{0}}}{\partial w}\right|_{\xi}\right|^{2}+O\left(N^{-1 / 2}\right)\right) .
$$

Similarly, if $d \phi_{z_{0}}(\xi)=0$, then

$$
\left\|\nabla_{\frac{\partial}{\partial z}}^{z_{0}} \otimes \bar{\nabla}_{\frac{\partial}{\partial z}}^{z_{0}} \Pi_{N}(w, w)\right\|_{h^{N}}=1+\left|\frac{\partial^{2} \phi_{z_{0}}}{\partial z^{2}}\right|_{\xi} \cdot w+\left.\bar{w}\right|^{2}+O\left(N^{-1 / 2}\right) .
$$

The local asymptotics (1.17) and (1.16) now follow by substituting the previous two expressions for $\left\|\nabla_{V}^{z_{0}} \otimes \overline{\nabla_{V}^{z_{0}}} \Pi_{N}\right\|$ into 7.2 and using 7.4. 


\section{Conditional Density of Critical Points: Proof of Theorem 3}

We mimic the proofs of Proposition 3.10 and Theorem 1.1 (in the $k=1$ case) in [16]. Let $h$ be a smooth positive Hermitian metric on $\mathcal{O}(1) \rightarrow \mathbb{C} P^{1}$. Fix $\xi \in \mathbb{C} P^{1}$ and define $H_{N}^{\xi}:=\left\{p_{N} \in H_{N} \mid p_{N}\left(z_{0}\right)=0\right\}$. As explained in Section 3 of [16], the distribution of $p_{N}$ conditional on $p_{N}(\xi)=0$ is the restriction of the Gaussian measure on $H_{h o l}^{0}\left(\mathbb{C} P^{1}, \mathcal{O}(N)\right)$ according to which $p_{N}$ is distributed to $H_{N}^{\xi}$.

Of Theorem 3. The key to proving the local and global asymptotics is the following result, which is the analog of Proposition 3.10 in [16].

Lemma 4. For each $N$,

$$
\left\|\nabla_{V}^{z_{0}} \otimes \overline{\nabla_{V}^{z_{0}}} \Pi_{N}^{\xi}(w, w)\right\|_{h^{N}}=\left\|\nabla_{V}^{z_{0}} \otimes \overline{\nabla_{V}^{z_{0}}} \Pi_{N}(w, w)\right\|_{h^{N}}\left(1-\widetilde{P}_{N}(\xi, w)^{2}\right),
$$

where

$$
\widetilde{P}_{N}(\xi, w)=\frac{\left\|1 \otimes \overline{\nabla_{V}^{z_{0}}} \Pi_{N}(\xi, w)\right\|_{h}}{\left\|\nabla_{V}^{z_{0}} \otimes \overline{\nabla_{V}^{z_{0}}} \Pi_{N}(w, w)\right\|_{h}^{1 / 2} \cdot\left\|\Pi_{N}(\xi, \xi)\right\|_{h}^{1 / 2}}
$$

is the correlation between $p_{N}(\xi)$ and $\nabla_{V}^{z_{0}} p_{N}(w)$ (cf Section 3).

Proof. Choose $e_{\xi}^{*} \in \mathcal{O}(N)_{\xi}^{*}$ a unit dual vector. Define the "coherent state" at $p_{N}(\xi)=0$

$$
\Psi_{N}^{\xi}(w):=\frac{1}{\left\|\Pi_{N}(\xi, \xi)\right\|^{1 / 2}} \iota_{\xi}^{*} \Pi_{N}(\xi, w) \in H_{N},
$$

where $\iota$ is the contraction operator. More explicitly, if $\left\{S_{N}^{j}, j=0, \ldots, N\right\}$ is any orthonormal basis for $H_{N}$ with

$$
S_{N}^{j}(w)=f_{j}(w) \cdot e_{N}(w)
$$

relative to a local holomorhpic frame $e_{N}$ for $\mathcal{O}(N)$, then

$$
\Psi_{H}^{\xi}(w)=\frac{1}{\left(\sum_{j=0}^{N}\left|f_{j}(\xi)\right|^{2}\right)^{1 / 2}} \sum_{j=0}^{N} \overline{f_{j}(\xi)} \cdot S_{j}(w) .
$$

Note that $\left\langle s, \Psi_{N}^{\xi}\right\rangle=0$ for each $s \in H_{N}^{\xi}$. Hence, $\Psi_{N}^{z_{0}}$ spans $H_{N}^{z_{0} \perp}$. Further, $\left\|\Psi_{N}^{\xi}\right\|_{h}=1$. So

$$
\left\|\nabla_{V}^{z_{0}} \otimes \overline{\nabla_{V}^{z_{0}}} \Pi_{N}^{\xi}(w, w)\right\|_{h}=\left\|\nabla_{V}^{z_{0}} \otimes \overline{\nabla_{V}^{z_{0}}} \Pi_{N}(w, w)\right\|_{h}-\left\|\nabla_{V}^{z_{0}} \Psi_{N}^{\xi}(w)\right\|_{h}^{2} .
$$

Noting that

completes the proof.

$$
\left\|\nabla_{V}^{z_{0}} \Psi_{N}^{\xi}(w)\right\|_{h}^{2}=\frac{\left\|1 \otimes \overline{\nabla_{V}^{z_{0}}} \Pi_{N}(\xi, w)\right\|_{h^{N}}^{2}}{\left\|\Pi_{N}(\xi, \xi)\right\|_{h^{N}}}
$$

We will first prove the local scaling asymptotics $(1.11)-(1.13)$. Fix $\zeta \in \mathbb{C} P^{1}$ an a $N^{-1 / 2}$-scaled normal coordinate centered at $\zeta$. Combining Corollary 3 and Lemma 4 , we may write

$$
E\left[C_{p_{N}} \mid p_{N}(\xi)=0\right](w)-E\left[C_{p_{N}}\right](w)=\frac{i}{2 \pi} \partial \bar{\partial} \log \left(1-\widetilde{P}_{N}(\xi, w)\right) .
$$


If $\zeta \neq \xi$, then by (5.2) $\widetilde{P}_{N}(\xi, w)=O\left(N^{-k}\right)$ for any $k \geq 1$, confirming 1.11$)$. Next, if $\zeta=\xi$, then sustituting (5.3) and (5.4) into $(8.2)$ proves $(1.12)$ and $(1.13)$.

To prove the global asymptotics 1.10 , we fix $\psi \in C^{2}\left(\mathbb{C} P^{1}\right)$ and seek to show that

$$
\int_{\mathbb{C} P^{1}} \psi(w)\left[E\left[C_{p_{N}} \mid p_{N}(\xi)=0\right](w)-E\left[C_{p_{N}}\right](w)\right]=O(1) .
$$

Using (8.2), this is equivalent to

$$
\int_{\mathbb{C} P^{1}} \psi(w) \frac{i}{2 \pi} \partial \bar{\partial} \log \left(1-\widetilde{P}_{N}(\xi, w)^{2}\right)=O(1) .
$$

Recall from $\sqrt{5.2}$ that $\widetilde{P}_{N}(\xi, w)=O\left(N^{-k}\right)$ for $|w-\xi| \geq\left(\frac{\log N}{N}\right)^{1 / 2}$. Hence, taking a Kähler normal coordinate centered at $\xi$, we may write (8.3) as

$$
\int_{|\xi-w| \leq\left(\frac{\log N}{N}\right)^{1 / 2}} \psi(w) \frac{i}{2 \pi} \partial \bar{\partial} \log \left(1-\widetilde{P}_{N}(\xi, w)^{2}\right)+O\left(N^{-k}\right)
$$

The local asymptotics (1.12) and (1.13) that we just proved show in particular that the near-diagongal integral is $O(1)$. This concludes the proof.

\section{Joint Density of Zeros and Critical Points: Proof of Theorem 1}

Fix $N \geq 1$ and $h$, a smooth positive Hermitian metric on $\mathcal{O}(N) \rightarrow \mathbb{C} P^{1}$. Let $p_{N} \in$ $H_{\text {hol }}^{0}\left(\mathbb{C} P^{1}, \mathcal{O}(N)\right)$ be drawn from the Hermitian Gaussian ensemble corresponding to $h$. We seek to compute the local and global asymptotics of the covariance current between the zeros and critical points of $p_{N}$ :

$$
\operatorname{Cov}_{N}(z, w)=E\left[Z_{p_{N}} \wedge C_{p_{N}}\right](z, w)-E\left[Z_{p_{N}}\right](z) \wedge E\left[C_{p_{N}}\right](w)
$$

As in [1, 15], we introduce

$$
G(t):=\frac{\gamma^{2}}{4}-\frac{1}{4} \int_{0}^{t^{2}} \frac{\log (1-s)}{s} d s, \quad 0 \leq t<1
$$

and recall the following

Lemma 5 (Lemma 3.3 from [15]). Let a be a standard Gaussian random vector in $\mathbb{C}^{N+1}$ and let $u, v \in C^{N+1}$ denote unit vectors. Then

$$
E[\log |\langle a, u\rangle| \log |\langle a, v\rangle|]=G(|\langle u, v\rangle|),
$$

where $\langle\cdot, \cdot\rangle$ is the usual Hermitian inner product on $\mathbb{C}^{N+1}$.

Our first step, Lemma 6, gives in the terminology of [15] a pluri bi-potential for $\operatorname{Cov}_{N}$. This result will be the starting point for proving both the local and global asymptotics of $\operatorname{Cov}_{N}$.

Lemma 6. Let $U \subseteq \mathbb{C} P^{1}$. In coordinates on $U$, we have

$$
\operatorname{Cov}_{N}(z, w)=\left(\frac{i}{\pi} \partial_{z} \bar{\partial}_{z}\right)\left(\frac{i}{\pi} \partial_{w} \bar{\partial}_{w}\right) G\left(\widetilde{P}_{N}(z, w)\right),
$$

where $\widetilde{P}_{N}$ is the absolute value of the correlation kernel between zeros and critical points:

$$
\widetilde{P}_{N}(z, w)=\frac{\left\|1 \otimes \overline{\nabla_{V}^{z_{0}}} \Pi_{N}(z, w)\right\|_{h^{N}}}{\sqrt{\left\|\Pi_{N}(z, z)\right\|_{h^{N}}\left\|\nabla_{V}^{z_{0}} \otimes \bar{\nabla}_{V}^{z_{0}} \Pi_{N}(w, w)\right\|_{h^{N}}}} .
$$


As elsewhere, $V$ is any auxiliary non-vanishing local holomorphic vector field on which $\widetilde{P}_{N}$ does not depend.

Proof. Denote by $e$ a frame for $\mathcal{O}(1)$ over $U \subseteq \mathbb{C} P^{1}$. Recall that $p_{N}(z)=\sum_{j=0}^{N} a_{j} S_{j}(z)$ for $a_{j}$ independent standard complex Gaussians and $\left\{S_{j}\right\}$ and orthonormal basis for 1.1 . We will write

$$
S_{j}^{N}=f_{j} \cdot e^{\otimes N}, \quad \nabla_{V} S_{j}^{N}=g_{j} \cdot e^{\otimes N}
$$

and abbreviate $a=\left[a_{0}, \ldots, a_{N}\right]$ as well as $f=\left[f_{0}, \ldots, f_{N}\right], g=\left[g_{0}, \ldots, g_{N}\right]$. Denoting by $\langle\cdot, \cdot\rangle$ the usual Hermitian inner product on $\mathbb{C}^{N+1}$, we have

$$
p_{N}(z)=\langle a, \bar{f}\rangle \cdot e^{\otimes N}, \quad \nabla_{V} p_{N}(z)=\langle a, \bar{g}\rangle \cdot e^{\otimes N} .
$$

For any smooth test fuction $\psi \in C^{\infty}\left(\mathbb{C} P \times \mathbb{C} P^{1}\right)$ we apply the Poincare-Lelong formula of Lemma 3 to write $\int_{U \times U} E\left[Z_{p_{N}} \wedge C_{p_{N}}\right](z, w) \psi(z, w)$ as

$$
E\left[\int_{U \times U} \frac{i}{\pi} \partial_{z} \bar{\partial}_{z} \log |\langle a, \overline{f(z)}\rangle| \frac{i}{\pi} \partial_{w} \bar{\partial}_{w} \log |\langle a, \overline{g(w)}\rangle| \psi(z, w)\right] .
$$

We now integrate by parts and, using that the resulting integrand is in $L^{1}$, exchange the order of integration to find

$$
E\left[Z_{p_{N}} \wedge C_{p_{N}}\right](z, w)=\left(\frac{i}{\pi} \partial_{z} \bar{\partial}_{z} \wedge \frac{i}{\pi} \partial_{w} \bar{\partial}_{w}\right) E[\log |\langle a, f(z)\rangle| \log |\langle a, g(w)\rangle|] .
$$

Just as in Section 3 of [1], we introduce $u(z):=\frac{\overline{f(z)}}{\|f(z)\|}, v(z):=\frac{\overline{g(w)}}{\|g(w)\|}$, to write

$$
E\left[C_{p_{N}} \wedge Z_{p_{N}}\right](z, w)=-\frac{1}{\pi^{2}}\left(E_{1}(z, w)+E_{2}(z, w)+E_{3}(z, w)+E_{4}(z, w)\right),
$$

where

$$
\begin{aligned}
& E_{1}(z, w):=\partial_{z} \bar{\partial}_{z} \partial_{w} \bar{\partial}_{w} \log \|f(z)\| \log \|g(w)\| \\
& E_{2}(z, w):=\partial_{z} \bar{\partial}_{z} \partial_{w} \bar{\partial}_{w} E[\log |\langle a, u(z)\rangle| \cdot \log \|g(w)\|] \\
& E_{3}(z, w):=\partial_{z} \bar{\partial}_{z} \partial_{w} \bar{\partial}_{w} E[\log |\langle a, v(w)\rangle| \cdot \log \|f(z)\|] \\
& E_{4}(z, w):=\partial_{z} \bar{\partial}_{z} \partial_{w} \bar{\partial}_{w} E[\log |\langle a, u(z)\rangle| \log |\langle a, v(w)\rangle|] .
\end{aligned}
$$

As in the proof of Theorem $4, E_{2}$ and $E_{3}$ vanish as they are independent of $z, w$, respectively (see also Section 3.2 of [12]). Further, as $\|f(z)\|=\sum_{j=0}^{N}\left|f_{j}(z)\right|^{2}$ and $\|g(w)\|=$ $\sum_{j=0}^{N}\left|\frac{\partial f_{j}}{\partial w}(w)\right|^{2}$, we see from Corollary 3 and Lemma 2 that

$$
-\frac{1}{\pi^{2}} E_{1}(z, w)=E\left[Z_{p_{N}}\right](z) \wedge E\left[C_{p_{N}}\right](w) .
$$

Hence, $-\frac{1}{\pi^{2}} E_{4}(z, w)=\operatorname{Cov}_{N}(z, w)$ and by Lemma 5

$$
E_{4}(z, w)=\partial_{z} \bar{\partial}_{z} \partial_{w} \bar{\partial}_{w} G(|\langle u(z), v(w)\rangle|) .
$$

Observing that $|\langle u(z), v(w)\rangle|=\widetilde{P}_{N}(z, w)$ completes the proof. 
The local asymptotics (1.5) follow immediately by substituting into equation (9.1) the asymptotic expansions (5.3) and (5.4). To prove the global asymptotics (1.5), it suffices by Lemma 6 to show that for any test function $\psi \in C^{\infty}\left(\mathbb{C} P^{1} \times \mathbb{C} P^{1}\right)$ and any $\epsilon>0$ that

$$
\int_{\mathbb{C} P^{1} \times \mathbb{C} P^{1}} G\left(\widetilde{P}_{N}(z, w)\right) \partial_{z} \bar{\partial}_{z} \partial_{w} \bar{\partial}_{w} \psi(z, w)=O\left(N^{\epsilon}\right) .
$$

Recall from 5.6 and 5.7) that $\widetilde{P}_{N}(z, w)<1$ for each fixed $N$. Since $G(t)$ is a smooth away from $t=1$, we see that $G\left(\widetilde{P}_{N}(z, w)\right)$ is smooth. Moreover, $G(t)$ is stictly increasing in $t$ and the singularity at $t=1$ is given by $G(1-t) \sim \log (t)$. Thus, since the weakest estimate (5.7) holds for all $z, w$ we see that

$$
G\left(\widetilde{P}_{N}(z, w)\right)=O(\log N)=O\left(N^{\epsilon}\right),
$$

allowing us to conclude $(9.6)$.

\section{Expected Nearest Neighbor Spacings: Proof of Theorem 2}

Fix $N \geq 1, \epsilon>0$, and $\xi \in \mathbb{C} P^{1}$. In $N^{-1 / 2}$-scale normal coordinates centerd at $\xi$, we fix $A \subseteq \mathbb{C}$ measurable with finite area. Our goal is to estimate

$$
E\left[\mathcal{X}_{N, A, \epsilon}\right]=\int_{\mathbb{C}^{2}} I_{w \in A}(w) \cdot I_{|z-w| \leq N^{-1 / 4+\epsilon}}(z, w) E\left[Z_{p_{N}} \wedge C_{p_{N}}\right](z, w)
$$

when $d \phi_{z_{0}}(\xi) \neq 0$ or $\xi=\infty$ and

$$
\int_{\mathbb{C}^{2}} I_{w \in A}(w) \cdot I_{|z-w| \in\left[|\zeta|^{-1} \pm|\zeta|^{-1-c}\right], \arg (z-w) \in\left[\arg (\zeta) \pm|\zeta|^{-c}\right]}(z, w) E\left[Z_{p_{N}} \wedge C_{p_{N}}\right](z, w)
$$

when $d \phi_{z_{0}}(\xi)=0$. By definition of $\operatorname{Cov}_{N}$,

$$
E\left[Z_{p_{N}} \wedge C_{p_{N}}\right](z, w)=\operatorname{Cov}_{N}(z, w)+E\left[Z_{p_{N}}\right](z) \wedge E\left[C_{p_{N}}\right](w) .
$$

From Theorems 1 and 4 , recall that

$$
E\left[Z_{p_{N}}\right](z)=\frac{i}{2 \pi} \partial_{z} \bar{\partial}_{z}|z|^{2}+O\left(N^{-1 / 2+\epsilon}\right)
$$

while

$$
E\left[C_{p_{N}}\right](w)=\frac{i}{2 \pi} \partial_{w} \bar{\partial}_{w}|w|^{2}+O\left(N^{-1 / 2+\epsilon}\right)
$$

when $d \phi_{z_{0}}(\xi) \neq 0$ or $\xi=\infty$ and, writing $\zeta=\left.\frac{\partial^{2} \phi_{z_{0}}}{\partial \bar{w}^{2}}\right|_{\xi} \bar{w}+w$,

$$
E\left[C_{p_{N}}\right](w)=\frac{i}{2 \pi} \partial_{w} \bar{\partial}_{w}|w|^{2}+\frac{i}{2 \pi} \partial_{w} \bar{\partial}_{w} \log \left(1+|\zeta|^{2}\right)+O\left(N^{-1 / 2+\epsilon}\right)
$$

if $d \phi_{z_{0}}(\xi)=0$. Hence, the contribution of $E\left[Z_{p_{N}}\right] \wedge E\left[C_{p_{N}}\right]$ to the integral 10.1$)$ is $O\left(N^{-1 / 4}\right)$. Similarly, the contibution to $\sqrt{10.2}$ is $O\left(N^{-1 / 2+\epsilon}\right)+O\left(\int_{A}|\zeta|^{-2}\right)$. To prove 1.7$)$ and 1.9 , we therefore focus on estimating the integrals (10.1) and (10.2) with $E\left[C_{p_{N}} \wedge Z_{p_{N}}\right]$ replaced by $\operatorname{Cov}_{N}(z, w)$. We do this by using the bi-potential obtained in Lemma 6 .

$$
\operatorname{Cov}_{N}(z, w)=\left(\frac{i}{\pi} \partial_{z} \bar{\partial}_{z} \wedge \frac{i}{\pi} \partial_{w} \bar{\partial}_{w}\right) G\left(\widetilde{P}_{N}(z, w)\right) .
$$


10.1. Case $1\left(d \phi_{z_{0}}(\xi) \neq 0\right)$. We begin with the case $d \phi_{z_{0}}(\xi) \neq 0$ or $\xi=\infty$.

Lemma 7. For each $N$ and $A$ and for some $R>0$,

$$
E\left[\mathcal{X}_{N, A}\right]=\frac{1}{\pi^{2}} \int_{z \in A} \int_{|z-w| \leq N^{-1 / 4}} \partial_{w} \bar{\partial}_{w}\left[\log \left(|z-w|^{2}+R \cdot N^{-1 / 2}\right)+O(1) .\right]
$$

Proof. Recall that $G(t)=\frac{\gamma^{2}}{2}-\frac{1}{4} \int_{0}^{t^{2}} \frac{\log (1-s)}{s} d s$ for $0 \leq t<1$. Thus,

$$
\partial_{z} \bar{\partial}_{z} G\left(\widetilde{P}_{N}(z, w)\right)=\partial_{z}\left[\frac{\log \left(1-\widetilde{P}_{N}(z, w)^{2}\right)}{\widetilde{P}_{N}(z, w)^{2}} \cdot \bar{\partial}_{z}\left(\widetilde{P}_{N}(z, w)^{2}\right)\right]
$$

Using (5.8)-(5.10), we may rewrite this as

$$
\begin{aligned}
\partial_{z} \bar{\partial}_{z} G\left(\widetilde{P}_{N}(z, w)\right) & =\frac{1}{\pi^{2}} \partial_{z}\left[\log \left(1-\widetilde{P}_{N}(z, w)^{2}\right) \cdot \bar{\partial}_{z}\left(\widetilde{P}_{N}(z, w)^{2}\right)\right] \\
& =\frac{1}{\pi^{2}} \log \left(1-\widetilde{P}_{N}(z, w)^{2}\right) \cdot\left(\partial_{z} \bar{\partial}_{z}\right)\left(\widetilde{P}_{N}(z, w)^{2}\right)+\frac{\left|\partial_{z} \widetilde{P}_{N}(z, w)^{2}\right|^{2}}{1-\widetilde{P}_{N}(z, w)^{2}} \\
& =\frac{1}{\pi^{2}} \log \left(|z-w|^{2}+R \cdot N^{-1 / 2}+O\left(N^{-3 / 4+\epsilon}\right)\right)+O(1) .
\end{aligned}
$$

To understand 10.3 , we prove the following perturbation of Laplace's law: $\Delta \log |z|^{2}=\pi \cdot \delta_{0}$.

Lemma 8 (Perturbed Laplace Equation). Fix $\alpha>0$ and $\psi \in C_{c}^{0}(\mathbb{C})$. Then for any $\epsilon>0$ and constant $K>0$,

$$
\int_{|u| \leq \frac{\log N}{N^{\alpha}}}\left[\frac{i}{2} \partial_{u} \bar{\partial}_{u} \log \left(|u|^{2}+K \cdot N^{-\alpha}\right)\right] \psi(u)=\pi \psi(0)+O\left(N^{-\alpha+\epsilon}\right) .
$$

Proof. The proof is by direct computation. We have

$$
\frac{i}{2} \partial_{u} \bar{\partial}_{u} \log \left(|u|^{2}+K \cdot N^{-\alpha}\right)=\frac{K \cdot N^{-\alpha}}{\left(|u|^{2}+K \cdot N^{-\alpha}\right)^{2}} \frac{i}{2} d u \wedge d \bar{u}
$$

Hence, denoting by $I$ the left hand side of (10.4) and making the change of coordinates $u \mapsto u \cdot K^{1 / 2} \cdot N^{-\alpha / 2}$, we have

$$
\begin{aligned}
I & =\int_{|u| \leq \log N} \frac{1}{\left(1+|u|^{2}\right)^{2}} \psi\left(\frac{u}{N^{\alpha}}\right) \frac{i}{2} d u \wedge d \bar{u} \\
& =\pi \psi(0)+O\left(N^{-\alpha+\epsilon}\right),
\end{aligned}
$$

by the continuity of $\psi$ and the fact that $\frac{i}{2 \pi} \frac{1}{\left(1+|u|^{2}\right)^{2}} d u \wedge d \bar{u}$ is the volume 1 Fubini-Study measure.

For each fixed $z$, we make the change of variables $u=z-w$ in (10.3) and apply Lemma 8 , Recalling that $E\left[C_{A}\right]=\int_{A} \frac{i}{2 \pi} d w \wedge d \bar{w}+O\left(N^{-1 / 2}\right)$ completes the proof of Theorem 2 in this case. 
10.2. Case $2\left(d \phi_{z_{0}}(\xi)=0\right)$. We fix $\xi \in \mathbb{C} P^{1} \backslash\{\infty\}$ such that $d \phi_{z_{0}}(\xi)=0$ and take a $N^{-1 / 2}$-scale normal coordinate for $\omega_{h}$ centered at $\xi$. We then fix a bounded measurable set $A \subseteq \mathbb{C} \backslash\{|\zeta| \leq 1\}$ and a parameter $c \in(2 / 3,1)$. We've denoted $\alpha=\frac{\partial^{2} \phi_{z_{0}}}{\partial \bar{w}^{2}}(\xi)$ at in the statement of the Theorem, and we define

$$
\zeta:=\alpha \bar{w}+w, \quad \eta:=\alpha \bar{w}+z .
$$

The proof in this case is a long computation. For the reader's convenience we provide an outline.

Outline. Recall that $G(t)=\frac{\gamma^{2}}{4}-\frac{1}{4} \int_{0}^{t^{2}} \frac{\log (1-s)}{s} d s$. As explained in the beginning of the proof, we seek to compute

$$
\int_{\mathbb{C} \times \mathbb{C}} I_{w \in A}(w) \cdot I_{|z-w| \in\left[|\zeta|^{-1} \pm|\zeta|^{-1-c}\right], \arg (z-w) \in\left[\arg (\zeta) \pm|\zeta|^{-c}\right]}(z, w) \operatorname{Cov}_{N}(z, w) .
$$

Our first step is to recall from Lemma 6 that

$$
\operatorname{Cov}_{N}(z, w)=-\frac{1}{\pi^{2}} \partial_{z} \bar{\partial}_{z} \partial_{w} \bar{\partial}_{w} G\left(\widetilde{P}_{N}(z, w)\right) .
$$

Writing $\frac{i}{2} d w \wedge d \bar{w}$ for the usual lebesgue volume form we seek to evaluate

$$
\frac{4}{\pi} \cdot \int_{A}\left[\int_{\arg (\zeta)-|\zeta|^{-c}}^{\arg (\zeta)+|\zeta|^{-c}} \int_{|\zeta|^{-1}-|\zeta|^{-1-c}}^{|\zeta|^{-1}+|\zeta|^{-1-c}} \frac{\partial^{2}}{\partial z \partial \bar{z}} \frac{\partial^{2}}{\partial w \partial \bar{w}} G\left(\widetilde{P}_{N}\left(w+r e^{i t}, w\right) r d r d t\right] \frac{i}{2 \pi} d w \wedge d \bar{w}\right.
$$

Our second step is to use (5.3) to write

$$
\widetilde{P}_{N}(z, w)=\widetilde{P}_{\infty}(z, w)+O\left(N^{-1 / 2+\epsilon}\right)
$$

where

$$
\widetilde{P}_{\infty}(z, w)=\frac{|\eta|}{\sqrt{1+|\zeta|^{2}}} e^{-\frac{1}{2}|\eta-\zeta|^{2}},
$$

is the $N \rightarrow \infty$ limit of $\widetilde{P}_{N}(z, w)$. We have already seen in Corollary 2 that, for each $N$, $\widetilde{P}_{N}(z, w) \leq C<1$ for some universal constant $C$. Hence, since $G(t)$ is smooth away from $t=1$, the integrand in 10.5 becomes

$$
\frac{\partial^{2}}{\partial z \partial \bar{z}} \frac{\partial^{2}}{\partial w \partial \bar{w}} G\left(\widetilde{P}_{N}(z, w)\right)=\frac{\partial^{2}}{\partial z \partial \bar{z}} \frac{\partial^{2}}{\partial w \partial \bar{w}} G\left(\widetilde{P}_{\infty}(z, w)\right)+O\left(N^{-1 / 2+\epsilon}\right) .
$$

Our third step is to compute the four derivatives of $G$. It is necessary to do so since we seek to compute non-smooth statistics and hence we cannot integrate by parts onto the test function. To this end, we introduce as in Lemma 3.5 of [15]

$$
\lambda(z, w):=-\log \widetilde{P}_{\infty}(z, w)=\frac{1}{2}\left[|z-w|^{2}+\log \left(1+|\alpha \bar{w}+w|^{2}\right)-\log \left(|\alpha \bar{w}+z|^{2}\right)\right] .
$$

We further write for $\lambda \geq 0$

$$
F(\lambda):=G\left(e^{-\lambda}\right)=\frac{\gamma^{2}}{4}-\frac{1}{2} \int_{\lambda}^{\infty} \log \left(1-e^{-2 s}\right) d s .
$$

We will compute

$$
\frac{\partial^{2}}{\partial z \partial \bar{z}} \frac{\partial^{2}}{\partial w \partial \bar{w}} F(\lambda(z, w))
$$


It will turn out (cf $(10.9)$ ) that these four derivatives may be written as

$F^{4}(\lambda(z, w)) \cdot\left|\frac{\partial}{\partial z} \lambda(z, w)\right|^{2} \cdot\left|\frac{\partial}{\partial w} \lambda(z, w)\right|^{2}+F^{3}(\lambda(z, w)) \cdot\left|\frac{\partial}{\partial z} \lambda(z, w)-\frac{\partial}{\partial w} \lambda(z, w)\right|^{2}+\frac{1}{2} F^{2}(\lambda(z, w))$

plus a small error, where we've written $F^{j}$ for the $j^{\text {th }}$ derivative of $F$. In Lemmas 9 and 10 we obtain estimates on the derivatives of $F$ and $\lambda$ in the regime of the integration in (10.5). Finally, we combine these estimate and read off the desired answer.

Computations. We write $F^{j}$ for the $j^{\text {th }}$ derivative of $F$ and abbreviate

$$
\partial_{z}:=\frac{\partial}{\partial z} \lambda(z, w), \partial_{w}:=\frac{\partial}{\partial w} \lambda(z, w),\left(\partial_{z} \partial_{\bar{z}}\right):=\frac{\partial^{2}}{\partial z \partial \bar{z}} \lambda(z, w)
$$

and so on. We will need the following derivatives of $\lambda(z, w)$ :

$$
\begin{aligned}
\partial_{z} & =\frac{1}{2}\left(\bar{\eta}-\bar{\zeta}-\frac{1}{\eta}\right) & \left(\partial_{z} \partial_{\bar{z}}\right) & =\frac{1}{2} \\
\partial_{w} & =\frac{1}{2}\left(\frac{\bar{\alpha} \zeta+\bar{\zeta}}{1+|\zeta|^{2}}-\bar{\eta}+\bar{\zeta}-\frac{\bar{\alpha}}{\bar{\eta}}\right) & \left(\partial_{w} \partial_{\bar{w}}\right) & =\frac{1}{2}\left(1+O\left(|\zeta|^{-2}\right)\right) \\
\left(\partial_{z} \partial_{\bar{w}}\right) & =\left(\partial_{w} \partial_{\bar{z}}\right)=-\frac{1}{2}\left(1+O\left(|\zeta|^{-2}\right)\right), & \left(\partial_{z} \partial_{w}\right) & =\left(\partial_{\bar{z}} \partial_{\bar{w}}\right)=0 .
\end{aligned}
$$

We have supressed all the delta functions $\delta_{\eta}$ since we are interested in these derivatives when $|\zeta|>1$ and $|\zeta-\eta|$ is small. Using these explicit formuae, we have

$$
\frac{\partial^{2}}{\partial z \partial \bar{z}} \frac{\partial^{2}}{\partial w \partial \bar{w}}\left(G\left(\widetilde{P}_{\infty}\right)\right)=F^{4} \cdot\left|\partial_{z}\right|^{2} \cdot\left|\partial_{w}\right|^{2}+F^{3} \cdot \frac{1}{2}\left[\left|\partial_{w}-\partial_{z}\right|^{2}+O\left(|\zeta|^{-2}\right)\right]+F^{2} \cdot \frac{1}{4}\left[2+O\left(|\zeta|^{-2}\right)\right] .
$$

Next, we record the derivatives of $F$ evaluated at $\lambda>0$ :

$$
\begin{aligned}
F^{1}(\lambda) & =\frac{1}{2} \log \left(1-e^{-2 \lambda}\right), & F^{2}(\lambda) & =\frac{e^{-2 \lambda}}{1-e^{-2 \lambda}} \\
F^{3}(\lambda) & =-2 \cdot \frac{e^{-2 \lambda}}{\left(1-e^{-2 \lambda}\right)^{2}}, & F^{4}(\lambda) & =4 \cdot \frac{e^{-2 \lambda}+e^{-4 \lambda}}{\left(1-e^{-2 \lambda}\right)^{3}} .
\end{aligned}
$$

Next, in order to estimate the derivatives of $F$ and $\lambda$ in the region of interest for the integral (10.5), we write

and assume that

$$
z=w+r e^{i t} \quad \text { and } \quad r=\frac{1+\epsilon}{|\zeta|}
$$

$$
|\zeta|>1, \quad \text { and } \quad \epsilon \in\left[-|\zeta|^{-c},|\zeta|^{-c}\right], \quad \text { and } \quad t \in\left[\arg (\zeta)-|\zeta|^{-c}, \arg (\zeta)+|\zeta|^{-c}\right] \text {. }
$$

Observe that for this range of $z, w$ we have $\widetilde{P}_{\infty}(z, w)=1+O\left(|\zeta|^{-2}\right)$. Hence, the numerator in the above expression 10.11 for $F^{4}$ may be written

$$
4\left(e^{-4 \lambda}+e^{-2 \lambda}\right)=8 \widetilde{P}_{\infty}(z, w)^{2}\left(1+\left(|\zeta|^{-2}\right)\right) .
$$

The integrand in the integral 10.5 we seek to compute may be written as $(10.13)$

$$
\frac{4}{\pi^{2}} \cdot\left[\frac{\frac{1}{2}\left(1+O\left(|\zeta|^{-2}\right)\right) r d r \wedge d t}{1-\widetilde{P}_{\infty}(z, w)^{2}}\right]\left(\frac{16 \cdot\left|\partial_{z}\right|^{2} \cdot\left|\partial_{w}\right|^{2}}{\left(1-\widetilde{P}_{\infty}(z, w)^{2}\right)^{2}}-\frac{2 \cdot\left|\partial_{w}-\partial_{z}\right|^{2}}{1-\widetilde{P}_{\infty}(z, w)^{2}}+1\right) \frac{i}{2} d w \wedge d \bar{w}
$$


To evaluate 10.13 , we give estimates on $\left(1-\widetilde{P}_{\infty}(z, w)^{2}\right)^{-1}$ in Lemma 9 and on the various derivatives of $\lambda$ in Lemma 10.

Lemma 9. Write $r=\frac{1+\epsilon}{|\zeta|}$ and assume 10.12. We have the following expression for $\left(1-\widetilde{P}_{\infty}\left(w+r e^{i t}, w\right)^{2}\right)^{-1}:$

$$
\frac{|\zeta|^{4}\left(1+O\left(|\zeta|^{-c}\right)\right)}{\frac{1}{2}+(|\zeta| \epsilon)^{2}+(|\zeta|(t-\arg (\zeta)))^{2}}
$$

Proof. Recall that

$$
\widetilde{P}_{\infty}(z, w)^{2}=\frac{|\eta|^{2}}{1+|\zeta|^{2}} e^{-|\eta-\zeta|^{2}}
$$

This is invariant under rotation of $(\eta, \zeta)$. Hence, we may assume that $\zeta \in \mathbb{R}_{+}$and that $t \in\left[-|\zeta|^{-c},|\zeta|^{-c}\right]$. We write

$$
\left(1-\widetilde{P}_{\infty}(z, w)^{2}\right)^{-1}=\frac{\left(1+\zeta^{2}\right) e^{|\eta-\zeta|^{2}}}{\left(1+\zeta^{2}\right) e^{|\eta-\zeta|^{2}}-|\eta|^{2}}
$$

Using that $|\eta|^{2}=\zeta^{2}+r^{2}+2(1+\epsilon) \cos t$ and taylor expanding $e^{|\eta-\zeta|^{2}}$ and $\cos t$ we obtain (10.14.

Lemma 10. Fix $w$. Write $z=w+r e^{i t}$ and assume the regime (10.12). We have

$$
\left|\partial_{z} \lambda(z, w)\right|^{2}=\left|\partial_{w} \lambda(z, w)\right|^{2}=\frac{1}{4}\left|\partial_{z} \lambda(z, w)-\partial_{w} \lambda(z, w)\right|^{2}=\frac{\epsilon^{2}+(t-\arg (\zeta))^{2}}{4|\zeta|^{2}}+O\left(|\zeta|^{-2-3 c}\right) .
$$

Proof. Observe that $\frac{1}{\eta}-\frac{1}{\zeta}=O\left(|\zeta|^{-3}\right)$. Hence, from 10.6 ,

$$
\left|\partial_{z} \lambda(z, w)\right|^{2}=\frac{1}{4}\left|\bar{\eta}-\bar{\zeta}-\frac{1}{\zeta}+O\left(|\zeta|^{-3}\right)\right|^{2} .
$$

This expression is invariant under rotation of $(\eta, \zeta)$. Hence, we may assume that $\zeta \in \mathbb{R}_{+}$. The equation $\eta=\zeta+r e^{i t}$ then reads

$$
\eta=\zeta+\frac{1+\epsilon}{\zeta} e^{i t}
$$

Using that

$$
\left|(1+\epsilon)-e^{-i t}\right|^{2}=\epsilon^{2}+2(1+\epsilon)(1-\cos t)=\epsilon^{2}+t^{2}+O\left(\zeta^{-3 c}\right)
$$

allows us to conclude the desired expression for $\left|\partial_{z} \lambda(z, w)\right|^{2}$. To prove the same expression for $\left|\partial_{w} \lambda(z, w)\right|^{2}$, we observe that

$$
\frac{\bar{\alpha} \zeta}{1+|\zeta|^{2}}-\frac{\bar{\alpha}}{\bar{\eta}}=O\left(|\zeta|^{-3}\right)
$$

We then write

$$
\left|\partial_{w} \lambda(z, w)\right|^{2}=\frac{1}{4}\left|\frac{\zeta}{1+|\zeta|^{2}}+\zeta-\eta+O\left(|\zeta|^{-3}\right)\right|^{2}
$$


As before, this expression is under rotation of $(\eta, \zeta)$ so we may take $\zeta \in \mathbb{R}_{+}$. We then use that

$$
\frac{\zeta}{1+\zeta^{2}}-\frac{1}{\zeta}=O\left(\zeta^{-3}\right)
$$

and equations 10.16-10.17 to conclude the desired expression for $\left|\partial_{w} \lambda(z, w)\right|^{2}$. Finally,

$$
\left|\partial_{z} \lambda(z, w)-\partial_{w} \lambda(z, w)\right|^{2}=\left|2\left(\bar{\eta}-\bar{\zeta}-\frac{1}{\bar{\eta}}\right)-\frac{\zeta}{1+|\zeta|^{2}}+\frac{1}{\bar{\eta}}+O\left(|\zeta|^{-3}\right)\right| .
$$

This expression is invariant under rotation of $(\eta, \zeta)$. Taking $\zeta \in \mathbb{R}_{+}$, we see from (10.16) that $\bar{\eta}-\bar{\zeta}-\frac{1}{\bar{\zeta}}=(1+\epsilon) e^{-i t}-1$. Observing $-\frac{\zeta}{1+|\zeta|^{2}}+\frac{1}{\bar{\eta}}=O\left(|\zeta|^{-3}\right)$ and using 10.17 concludes the proof.

We define $D(r, t):=\frac{\epsilon^{2}+(t-\arg (\zeta))^{2}}{|\zeta|^{2}}$. Using Lemma 10 , we may write the integrand in the formula 10.13 for $E\left[\mathcal{X}_{A, N}\right]$ as

$$
\frac{\frac{1}{2}\left(1+O\left(|\zeta|^{-2}\right)\right) r d r \wedge d t}{1-\widetilde{P}_{\infty}\left(w+r e^{i t}, w\right)^{2}}\left(1-\frac{D(r, t)}{1-\widetilde{P}_{\infty}\left(w+r e^{i t}, w\right)^{2}}\right)^{2} .
$$

We now change variables in 10.19 from $(r, t)$ to $(x:=|\zeta| \cdot \epsilon, y:=|\zeta| \cdot(t-\arg (\zeta)))$. From the estimate (10.14), we have that

$$
\frac{\frac{1}{2}\left(1+O\left(|\zeta|^{-2}\right)\right) r d r \wedge d t}{1-\widetilde{P}_{\infty}(z, w)^{2}}=\frac{\frac{1}{2}\left(1+O\left(|\zeta|^{-1-c}\right)\right) d x \wedge d y}{\frac{1}{2}+x^{2}+y^{2}} .
$$

Similarly, combining 10.14 with 10.15), we find that

$$
\left(1-\frac{D(r, t)}{1-\widetilde{P}_{\infty}\left(w+r e^{i t}, w\right)^{2}}\right)^{2}=\frac{1}{4} \frac{1}{\left(\frac{1}{2}+x^{2}+y^{2}\right)^{2}} .
$$

Combining (10.14) and 10.15), we write the inner integral in 10.5 as

$$
\frac{1}{8} \int_{-|\zeta|^{1-c}}^{|\zeta|^{1-c}} \int_{-|\zeta|^{1-c}}^{|\zeta|^{1-c}} \frac{\left(1+O\left(|\zeta|^{-c}\right)+O\left(N^{-1 / 2+\epsilon}\right)\right) d x d y}{\left(1 / 2+x^{2}+y^{2}\right)^{3}}
$$

passing to polar coordinates we see that for $c<1$, this integral equals $\frac{\pi}{4}+O\left(|\zeta|^{2-3 c}\right)+$ $O\left(N^{-1 / 2+\epsilon}\right)$. Substituting this into 10.5 and recalling from 1.17 of Theorem 4 that $E\left[C_{A}\right]=$ $\int_{A} \frac{i}{2 \pi} d w \wedge d \bar{w}+O\left(\int_{A}|\zeta|^{-2}\right)+O\left(N^{-1 / 2+\epsilon}\right)$ completes the proof.

\section{REFERENCES}

[1] P. Bleher, B. Shiffman, and S. Zelditch. Poincare-lelong approach to universality and scaling of correlations between zeros. Commun. Math. Phys., 208(3):771-785, 2000.

[2] M. Douglas, B. Shiffman, and S. Zelditch. Critical points and supersymmetric vacua. Commun. Math. Phys., 252:325-358, 2004.

[3] M. Douglas and S. Zelditch. Asymptotics of almost holomorphic sections of ample line bundles on symplectic manifolds. J. reine angew, 544:181-222, 2002.

[4] M. Douglas and S. Zelditch. Critical points and supersymmetric vacua, ii: Asymptotics and extremal metrics. J. Diff. Geometry, 72:381-427, 2006.

[5] M. Douglas and S. Zelditch. Critical points and supersymmetric vacua, iii: String/m models. Commun. Math. Phys., 265:617-671, 2006.

[6] A. Edelman and E. Kostlan. How many zeros of a random polynomials are real? Bull. AMS, 32:1-37, 1995. 
[7] R. Feng and Z. Wang. Extrema of gaussian su(2) random polynomials. In Preparation.

[8] M. Krishnapur. Zeros of Random Analytic Functions. PhD thesis, U. C. Berkeley, 2006.

[9] B. Macdonald. Density of complex critical points of a real random so $(m+1)$ polynomial. Journal of Statistical Physics, 141(3):517-531, 2010.

[10] F. Nazarov and M. Sodin. Correlation functions for random complex zeros: Strong clustering and local universality. Commun. Math. Phys., 310(1):75-98, 2012.

[11] F. Nazarov, M. Sodin, and A. Volberg. Transportation to random zeroes by the gradient flow. Geom. Funct. Anal, 17(3):887-935, 2007.

[12] B. Shiffman and S. Zelditch. Distribution of zeros of random and quantum chaotic sections of positive line bundles. Commun. Math. Phys., 200:661-683, 1999.

[13] B. Shiffman and S. Zelditch. Asymptotics of almost holomorphic sections of ample line bundles on symplectic manifolds. J. reine angew, 544:181-222, 2002.

[14] B. Shiffman and S. Zelditch. Equilibrium distribution of zeros of random polynomials. Int. Math Res. Notices, pages 25-49, 2003.

[15] B. Shiffman and S. Zelditch. Number variance of random zeros on complex manifolds. Geom. Funct. Anal, 18(4):1422-1475, 2008.

[16] B. Shiffman, S. Zelditch, and Q. Zhong. Random zeros on complex manifolds: Conditional expectations. Journal of the Inst. of Math. Jussieu, 2011.

[17] S. Zelditch. Szego kernels and a theorem of tian. Int. Math Res. Notices, 6:317-331, 1998.

Department of Mathematics, Northwestern University, 2033 Sheridan Rd. Evanston, IL, 60208

E-mail address: bhanin@math.northwestern.edu 